\title{
Spatio-Temporal Model Reduction of Inverter-Based Islanded Microgrids
}

\author{
A THESIS \\ SUBMITTED TO THE FACULTY OF THE GRADUATE SCHOOL \\ OF THE UNIVERSITY OF MINNESOTA \\ BY
}

Ling Luo

IN PARTIAL FULFILLMENT OF THE REQUIREMENTS FOR THE DEGREE OF MASTER OF SCIENCE

Advisor: Sairaj V. Dhople

January, 2014 
(C) Ling Luo 2014

ALL RIGHTS RESERVED 


\section{Acknowledgements}

There are many people that have earned my gratitude for their contribution to my time in graduate school.

First, I owe my deepest gratitude to my advisor, Prof. Sairaj V. Dhople for his significant helps in my course studies and academic research. His broad knowledge, outstanding thoughts and passions to research impressed me deeply.

I am also grateful to the committee members of my master's thesis and instructors of the courses I have taken, Prof. Ned Mohan, Prof. Bruce Wollenberg and Prof. Peter Seiler Jr. Their valuable suggestions contributed significantly to this work.

Furthermore, I would like to thank my lab-mates Vivek Bhandari, Hyungjin Choi, Mohit Sinha, Ruben Otero-De-Leon and David Orser, my classmates Ahmed Ahmed, Andrew Stewart, Seema Deshpande, Nandini Ganesan, Yinan Wang, Xinyao Cheng and Mengying Ding. Thank you for all the helps and discussions.

Finally, I would like to thank all my friends at the University of Minnesota for the joyful days we spent together. 


\section{Dedication}

To my beloved husband, Guangyue $\mathrm{Xu}$, my son Leonardo L. Xu, my father Manren Luo, mother Donglian Luo, father-in-law Yingxi Xu and mother-in-law Meiying Zhang, for their endless and unconditional love and support. 


\begin{abstract}
Microgrids, are small foot-print power systems that balance critical loads against available energy supply, and are capable of operating in both grid-connected and islanded operation modes. Numerous factors such as energy assurance, reliability, renewable integration and economics are driving increased research and development in the modeling, analysis and control of microgrids.

In intentionally islanded operation, well-established droop control techniques are employed to keep inverters synchronized and regulate frequency and voltage within stability limits in microgrids. Computationally efficient and accurate models that describe droop-controlled inverter dynamics are key to controller design, stability assessment, and performance evaluation of islanded microgrids. Typical models for droop-controlled inverters are very detailed, and include myriad states from internal control loops and filters. Conceivably, control design, numerical simulations, and stability assessment with such models in islanded microgrids comprising tens of or even hundreds of inverters is computationally expensive and do not offer any analytical insights. This calls for the development of reduced-order models of inverter-based microgrids. Model reduction methods can isolate relevant spatio-temporal dynamics and mutual interactions of interest. While model reduction methods have been widely applied in bulk power systems, a systematic model-reduction procedure for droop-controlled islanded inverters has thus far been lacking. The objective of this thesis is to reduce large-signal dynamic models of inverter-based islanded microgrids in both spatial and temporal aspects. Singular perturbation methods are applied for temporal model reduction, and Kron reduction is employed for the spatial model reduction. The ensuing reduced-order models accurately describe the original dynamics with reduced computational burden. In addition, spatial model reduction isolates the mutual inverter interactions and clearly illustrates the equivalent loads that the inverters have to support in the microgrid - this aspect is leveraged in controller design to minimize power losses and voltage deviations.
\end{abstract}




\section{Contents}

\begin{tabular}{ll}
\hline Acknowledgements & i
\end{tabular}

Dedication $\quad$ ii

Abstract iii

$\begin{array}{ll}\text { List of Tables } & \text { vi }\end{array}$

List of Figures vii

\begin{tabular}{lll}
\hline 1 & Introduction & 1
\end{tabular}

\begin{tabular}{|lll}
\hline 2 & System Model & 4
\end{tabular}

2.1 Single Inverter Model $\ldots \ldots \ldots$. . . . . . . . . . . . . . . . . 4

2.2 Multiple-Inverter Model . . . . . . . . . . . . . . . . . . . 7

2.3 Network Model . . . . . . . . . . . . . . . . . . . . . . . . . . . . . . . . 8

\begin{tabular}{lll}
\hline 3 & Temporal Model Reduction & 10
\end{tabular}

3.1 Temporal Model Reduction Based on Singular Perturbation Methods . . 11

3.2 Reduced Fifth-Order Model . . . . . . . . . . . . . . . . . . . . . . . . . 12

3.3 Reduced Third-Order Model . . . . . . . . . . . . . . . . . . . . . . . . . 14

3.4 Reduced Single-Order Model . . . . . . . . . . . . . . . . . . . 15

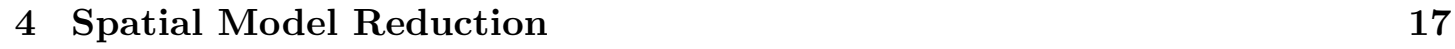

4.1 Kron Reduction . . . . . . . . . . . . . . . . . . . . . . . . . 17

4.2 Input of Inverter Dynamical Models $\ldots \ldots \ldots \ldots$ 
4.3 Software Package Developed in MATLAB . . . . . . . . . . . . . . . 19

\begin{tabular}{|lll}
\hline 5 & Numerical Studies & 21
\end{tabular}

5.1 Case 1: Six-Bus Microgrid . . . . . . . . . . . . . . . . . . . . . . 21

5.1 .1 Responses of Fast and Slow Dynamic Variables . . . . . . . . . . 22

5.1 .2 Original Model Compared to Reduced Models. . . . . . . . . . . 24

5.2 Case 2: IEEE 37-Bus Microgrid . . . . . . . . . . . . . . . . . . 33

$5.2 .1 \quad$ Original Model Compared to Reduced Models . . . . . . . . . . . 34

$5.2 .2 \quad$ Systematic Design of Droop Coefficients . . . . . . . . . . . . . . 41

5.3 Comparison of Simulation Speed Between the Full-order and the Reduced-

Order Models . . . . . . . . . . . . . . . . . . . . . . 46 46

6 Conclusions and Future Work 48

6.1 Conclusions . . . . . . . . . . . . . . . . . . . . . 48

6.2 Future Work . . . . . . . . . . . . . . . . . . . . . . . 49

\begin{tabular}{ll}
\hline References & 50
\end{tabular}

\begin{tabular}{|ll}
\hline Appendix A. Detailed Models & 54
\end{tabular}

A.1 Single-Order Model . . . . . . . . . . . . . . . . . . . . . . 54

A.2 Third-Order Model . . . . . . . . . . . . . . . . . . . . 55

A.3 Fifth-Order Model . . . . . . . . . . . . . . . . . . . . . 55

A.4 Ninth-Order Model . . . . . . . . . . . . . . . . . . . . . . . . 56

\begin{tabular}{ll}
\hline Appendix B. Simulation Parameters & 57
\end{tabular}

B.1 Six-Bus System . . . . . . . . . . . . . . . . 57

B.2 IEEE 37-Bus System . . . . . . . . . . . . . . . . . . . . 57 


\section{List of Tables}

5.1 Network parameters of Case 1 . . . . . . . . . . . . . . . . . . . 22

5.2 Maximum capacity of the inverters . . . . . . . . . . . . . . 43

5.3 Droop coefficients . . . . . . . . . . . . . . . . . . 43

5.4 Power losses and voltage deviations . . . . . . . . . . . . . . . . 43

5.5 Test of computational time in six-bus system . . . . . . . . . . . 46

5.6 Test of computational time in IEEE 37-bus system . . . . . . . . . . . . 46

B.1 Single inverter parameters . . . . . . . . . . . . . . . 57

B.2 IEEE 37-bus system branch parameters $\ldots \ldots \ldots \ldots \ldots$. . . . . . 58

B.3 IEEE 37-bus system branch parameters (continued) . . . . . . . . . . . 59

B.4 IEEE 37-bus system load parameters . . . . . . . . . . . . . . . . . 60 


\section{List of Figures}

2.1 The principle of droop control: (a)voltage droop; (b)frequency droop. . $\quad 4$

2.2 Block diagram of the controller for a single inverter . . . . . . . . . . 5

2.3 Multiple inverters integrated to microgrid: (a) block diagram; (b) angle reference frames. . . . . . . . . . . . . . . . . . 7

2.4 Oneline diagram of a sample microgrid with three inverters (inverters are denoted by solid blue dots). . . . . . . . . . . . . . . . . . . . 99 9

$4.1 \quad$ Flow chart of dynamic simulations in MATLAB . . . . . . . . . . 20

5.1 Oneline diagram of microgrid with three inverters: (a) Original network; (b) Kron-reduced network (inverters are depicted with blue dots). . . . . . . . . . 21

5.2 Comparison of slow and fast dynamic variables. (a) Responses of the slow variables $\delta, P, Q$; (b) Responses of the fast variables $\phi_{d}, \phi_{q}, \gamma_{d}, \gamma_{q}$. $\quad 23$

5.3 Terminal voltage angle transients from startup comparing the original 13th-order and the spatio-temporal reduced models: $(\mathrm{a}) \delta_{2} ;(\mathrm{b}) \delta_{3} . \ldots 25$

5.4 Active power transients from startup comparing the original 13th-order and the spatio-temporal reduced models: (a) $P_{1} ;(\mathrm{b}) P_{2} . . \ldots 26$

$5.5 \quad$ Active and Reactive power transients from startup comparing the original 13th-order and the spatio-temporal reduced models: (a) $P_{3} ;$ (b) $Q_{1}$. . . . 27

$5.6 \quad$ Reactive power transients from startup comparing the original 13th-order and the spatio-temporal reduced models: $(\mathrm{a}) Q_{2} ;(\mathrm{b}) Q_{3} . \ldots 28$

5.7 Terminal voltage angle transients from startup comparing the original 13th-order and the spatio-temporal reduced models: $(\mathrm{a}) \delta_{2} ;(\mathrm{b}) \delta_{3}$. . . . . 29

5.8 Active power transients from startup comparing the original 13th-order and the spatio-temporal reduced models: $(\mathrm{a}) P_{1} ;(\mathrm{b}) P_{2} . \ldots \ldots 30$ 
$5.9 \quad$ Active and Reactive power transients from startup comparing the original 13th-order and the spatio-temporal reduced models: (a) $P_{3} ;$ (b) $Q_{1}$. . . . 31

5.10 Reactive power transients from startup comparing the original 13th-order

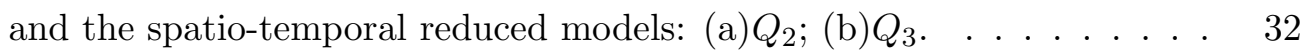

5.11 One-line diagram of modified IEEE 37-bus test system (inverters are represented by blue dots). . . . . . . . . . . . . . . 33

5.12 Step responses of terminal voltage angles of seven inverters. . . . . . . . 34

5.13 Step responses of active power of seven inverters. . . . . . . . . . . . . . 35

5.14 Step responses of reactive power of seven inverters. . . . . . . . . . . . . 35

5.15 Step responses of terminal voltage angle comparing the original 13th-order and the spatio-temporal reduced models: $(\mathrm{a}) \delta_{2} ;(\mathrm{b}) \delta_{3} ;(\mathrm{c}) \delta_{7}$. . . . . . . . . . . . . 36

5.16 Step responses of active power comparing the original 13th-order and the spatio-temporal reduced models: $(\mathrm{a}) P_{1} ;(\mathrm{b}) P_{2} . \ldots . \ldots 37$

5.17 Step responses of active power comparing the original 13th-order and the spatio-temporal reduced models: $(\mathrm{a}) P_{3} ;(\mathrm{b}) P_{7} \ldots \ldots . \ldots 38$

5.18 Step responses of reactive power comparing the original 13th-order and the spatio-temporal reduced models: $(\mathrm{a}) Q_{1} ;(\mathrm{b}) Q_{2} \ldots$. . . . . . . 39

5.19 Step responses of reactive power comparing the original 13th-order and the spatio-temporal reduced models: $(\mathrm{a}) Q_{3} ;(\mathrm{b}) Q_{7} \ldots$. . . . . . . . 40

5.20 Oneline diagram of IEEE 37-bus system after Kron reduction . . . . . . 41

5.21 Scenario 2: step responses of terminal voltage angles of seven inverters. 44

5.22 Scenario 2: step responses of active power of seven inverters. . . . . . . 45

5.23 Scenario 2: step responses of reactive power of seven inverters. . . . . . 45 


\section{Chapter 1}

\section{Introduction}

Numerous factors such as energy assurance, reliability, renewable integration, and economics are driving increased research and development in the modeling, analysis, and control of microgrids [1, 2, 3, 4, To assure the stability and reliability of the microgrids, it is important to build dynamical models for microgrids that clearly capture the interaction of the loads, sources, and power converter circuits.

A microgrid can be operated either in grid-connected mode or islanded mode. In islanded operation mode, the microgrid can balance critical loads against available energy supply while maintaining frequency and voltage within allowed limits by droop controllers. Droop controllers are designed to mimic the synchronous machines in conventional power system. Typical models for droop-controlled inverters are very detailed, and include myriad states from internal control loops and filters [4, 5, 6, 7, 8]. Conceivably, control design, numerical simulations, and stability assessment with such models in islanded microgrids comprising tens of or even hundreds of inverters is computationally expensive and do not offer any analytical insights. This calls for the development of model-reduction methods to isolate relevant spatio-temporal dynamics and mutual interactions of interest. While model reduction methods have been widely applied in bulk power systems, a systematic model-reduction procedure for droop-controlled islanded inverters has thus far been lacking. To fill the blank, a systematic model reduction method is proposed in this thesis to reduce large-signal dynamic models of inverterbased islanded microgrids in both spatial and temporal aspects.

Model reduction techniques for dynamic models of bulk power system are well developed. 
Examples of methods include Krylov subspace methods [9], Gramian-based method [10], aggregate slow coherency [11], and singular perturbation methods [12, 13, 14].

Moment matching methods built on Krylov subspace in [9] reduced power system order by projecting the linearized model to Krylov subspace while retaining information about important moments. Gramian-based method in [10] is proposed to efficiently reduce linear systems by computing approximations to the controllability and observability gramians of large sparse power system descriptor models. Aggregate slow coherency methods used in bulk power system analysis, simulations and islanding strategy studies [15, 16] are implemented with steps of linearization, calculation of eigenvalues and eigenvectors, and division of coherent groups. Singular perturbation methods provide a systematic approach to multiple time scales modeling (fast dynamic variables and slow dynamic variables), analysis and control of dynamic systems with widely different speeds [17, 18, 19]. In singular perturbation methods, reduced-order models are obtained by simply neglecting dynamics of the fast variables [12, 14, 18, 20].

Compared to Krylov subspace methods, Gramian-based model reduction methods, and slow coherency methods which require linearization and calculation of eigenvalues (or even eigen-vectors), singular perturbation methods are much easier and effective for dynamic models composed of differential equations with multiple physical small parameters. A typical large-signal dynamic model of an inverter-based islanded microgrid described in [4, 8] includes a power controller (within which droop control laws are embedded), as well as additional loops for voltage and current control. The model includes differential equations with multiple physical small parameters which motivates the application of singular-perturbation methods. Consequently, a temporal model reduction based on singular perturbation methods is proposed for droop-controlled inverters.

On the other hand, Kron reduction, proposed by Gebrial Kron in 1953 [21, is a standard model reduction tool employed in power networks for applications such as transient stability assessment [22, 23]. Here, Kron reduction is utilized to reduce the network in spatial aspect by eliminating internal, non-inverter nodes.

Based on the proposed spatio-temporal model reduction methods, a typical ninth-order large-signal dynamic model of inverter-based islanded microgrid is reduced to fifth-, third-, and single-order models in a step-by-step fashion in this thesis.

Microgrids are resistive dominant networks, and the power losses cannot be ignored. 
Based on the Kron-reduced network as is typically done in bulk power systems, the equivalent loads that the inverters have to support are computed. A systematical design strategy based on locally balancing equivalent loads against energy supply is proposed to design the inverters. We demonstrate that this design strategy minimizes power losses and voltage deviations in the network.

The fundamental idea of model reduction is that the input-output behaviors can be well approximated by a much simpler model. Thus, the computational efficiency and accuracy of the reduced-order models are tested in a MATLAB software package developed by the author. The remainder of this thesis is organized as follows:

- Chapter 2 introduces the large-signal dynamic model of an inverter-based islanded microgrid, including the model of a single inverter, multiple inverters and the electrical network.

- Chapter 3 presents the temporal model reduction based on singular perturbation methods, and successively reduces a ninth-order model to fifth-, third- and singleorder models step by step.

- Chapter 4 describes spatial model reduction method based on Kron reduction, integration of multiple inverters to microgrid, and introduces the functionality of the microgrid simulation software package developed in MATLAB .

- Chapter 5 includes numerical studies of spatio-temporal reduced models in an illustrative six-bus example adopted from the literature, as well as a modified IEEE 37-bus network.

- Chapter 6 concludes the thesis, and highlights some avenues for future work. 


\section{Chapter 2}

\section{System Model}

In this chapter, the system model of inverter-based islanded microgrids is described. We start from the model of single droop-controlled inverter, then move on to the model of microgrids composed of multiple inverters, and finally the network model.

\subsection{Single Inverter Model}

A microgrid can operate in both grid-connected mode and islanded mode. In case islanded operation mode, frequency and voltage are maintained by a primary control strategy based on active power and reactive power droop control [4, 7, 8, 24, 25, 26] (usually embedded in the power controller). In particular, the voltage and frequency of inverters is regulated according to Fig. 2.1. The active power $P$ droop control is used to reduce the frequency when the active power of loads increases; reactive power $Q$ droop control is used to reduce the voltage magnitude when reactive power of loads increases.

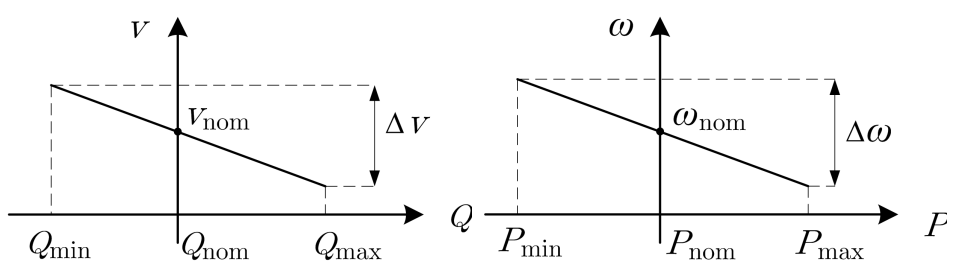

(a)

(b)

Figure 2.1: The principle of droop control: (a)voltage droop; (b)frequency droop. 
In Fig. 2.1. $V_{\text {nom }}\left(\omega_{\text {nom }}\right)$ is the nominal magnitude of the terminal voltage (nominal inverter frequency) that the controllers try to regulate to, $Q_{\text {nom }}\left(P_{\text {nom }}\right)$ is the reactive power (active power) the inverter should output to support nominal terminal voltage (nominal frequency). $\Delta V(\Delta \omega)$ is the limit of voltage deviation (frequency deviation) controlled by the slope of the droop control. The slopes of the voltage-reactive power (frequency-active power) are defined as droop coefficients $n_{\mathrm{Q}}\left(m_{\mathrm{P}}\right)$ which are given by

$$
m_{\mathrm{P}}=\frac{\Delta \omega}{P_{\max }-P_{\min }}, \quad n_{\mathrm{Q}}=\frac{\Delta V}{Q_{\max }-Q_{\min }} .
$$

Fig. 2.2 shows a block diagram of the controller employed in each inverter. In addition to the power controller (within which the droop laws are embedded) there are additional inner control loops to regulate the inverter output current and terminal voltage.

Let $\omega$ denote the electrical frequency of the inverter, and let $\omega_{\text {com }}$ denote an adopted common electrical frequency (typically, set by a preassigned inverter in the microgrid). Then, we will define the terminal voltage angle $\delta$ as follows

$$
\frac{d \delta}{d t}=\omega-\omega_{\mathrm{com}},
$$

which captures the phase angle of the terminal voltage of the inverter with respect to the phase angle of the reference inverter.

Consequently, we can transform the terminal voltage and output current for each inverter from abc to dq coordinates, using the terminal voltage angle as the transformation angle $\delta+\omega_{\text {com }} t$ to obtain $v_{\mathrm{o}}:=v_{\mathrm{od}}+\mathrm{j} v_{\mathrm{oq}}$ and $i_{\mathrm{o}}:=i_{\mathrm{od}}+\mathrm{j} i_{\mathrm{oq}}$.

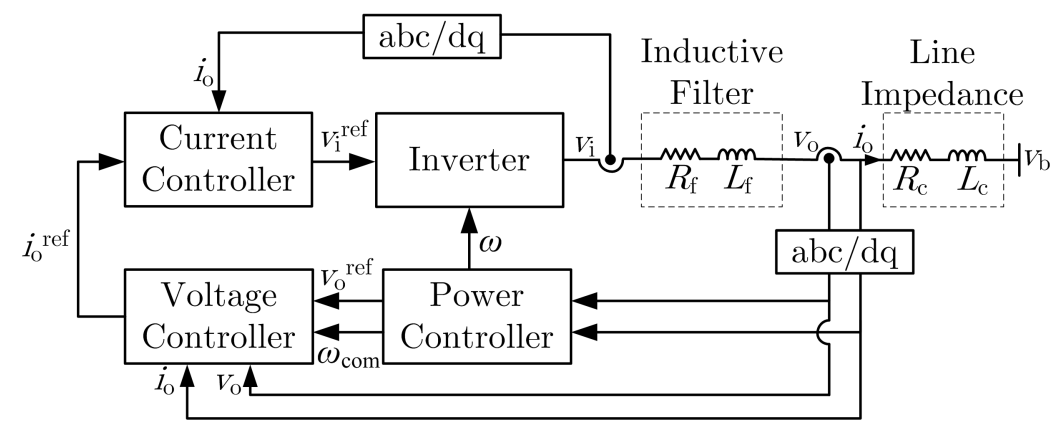

Figure 2.2: Block diagram of the controller for a single inverter

The dynamical equation that captures the operation of the power controller is given 
by:

$$
\frac{1}{\omega_{\mathrm{c}}} \frac{d S}{d t}=-S+v_{\mathrm{o}} i_{\mathrm{o}}^{*}
$$

where $\omega_{\mathrm{c}}$ is the cut-off frequency, and $S=P+\mathrm{j} Q$ is the apparent (low-pass filtered) power delivered by the inverter 1$]$ One output of the power controller is the terminalvoltage reference

$$
v_{\mathrm{o}}^{\mathrm{ref}}=v_{\text {nom }}-n_{\mathrm{Q}} \frac{S-S^{*}}{2}=v_{\mathrm{nom}}-n_{\mathrm{Q}} Q,
$$

which captures the voltage-reactive power droop law [4, 27, 24]. The other output, the inverter frequency, given by

$$
\omega=\omega_{\text {nom }}-m_{\mathrm{P}} \frac{S+S^{*}}{2}=\omega_{\text {nom }}-m_{\mathrm{P}} P,
$$

which is governed by the frequency-active power droop law [4, 27, 24].

The voltage- and current-controller state variables are denoted by $\phi=\phi_{\mathrm{d}}+\mathrm{j} \phi_{\mathrm{q}}$ and $\gamma=\gamma_{\mathrm{d}}+\mathrm{j} \gamma_{\mathrm{q}}$, respectively. Following [4, 5, 8, 28, a conventional PI controller is utilized to regulate the terminal voltage and output currents to their reference values, denoted by $v_{\mathrm{o}}^{\text {ref }}$ and $i_{\mathrm{o}}^{\text {ref }}$. The voltage- and current-controllers generate the references

$$
\begin{gathered}
i_{\mathrm{o}}^{\mathrm{ref}}=F i_{\mathrm{o}}+\mathrm{j} \omega+K_{\mathrm{p}}^{\phi} \frac{d \phi}{d t}+K_{\mathrm{i}}^{\phi} \phi, \\
v_{\mathrm{i}}^{\mathrm{ref}}=\mathrm{j} \omega_{\mathrm{nom}} L_{\mathrm{f}} i_{\mathrm{o}}+K_{\mathrm{p}}^{\gamma} \frac{d \gamma}{d t}+K_{\mathrm{i}}^{\gamma} \gamma,
\end{gathered}
$$

where $K_{\mathrm{p}}^{\phi}\left(K_{\mathrm{p}}^{\gamma}\right)$ and $K_{\mathrm{i}}^{\phi}\left(K_{\mathrm{i}}^{\gamma}\right)$ are the proportional (integral) parameters of the voltage (current) PI control blocks. The controller dynamics are governed by

$$
\begin{aligned}
& \frac{d \phi}{d t}=v_{\mathrm{o}}^{\mathrm{ref}}-v_{\mathrm{o}}, \\
& \frac{d \gamma}{d t}=i_{\mathrm{o}}^{\mathrm{ref}}-i_{\mathrm{o}} .
\end{aligned}
$$

Finally, the dynamics of the output current are captured by

$$
\frac{L_{\mathrm{c}}}{R_{\mathrm{c}}} \frac{d i_{\mathrm{o}}}{d t}=-\left(1+\mathrm{j} \omega \frac{L_{\mathrm{c}}}{R_{\mathrm{c}}}\right) i_{\mathrm{o}}+\frac{v_{\mathrm{o}}-v_{\mathrm{b}}}{R_{\mathrm{c}}}
$$

where $v_{\mathrm{b}}:=v_{\mathrm{bd}}+\mathrm{j} v_{\mathrm{bq}}$ is the bus voltage at the point of common coupling (PCC).

\footnotetext{
$1(\cdot)^{*}$ denotes complex conjugation.
} 


\subsection{Multiple-Inverter Model}

The large-signal dynamic model discussed above is based on individual dq reference frames for the inverters. In practical microgrids, there are usually more than one inverters integrated to the network shown in Fig. 2.3 (a). A common reference frame is adopted for the whole system, e.g. the reference frame of the first inverter. Letting the quadrature component of terminal voltage to zero for control purpose, and putting $v_{\mathrm{o}}$ on the direct axis of the corresponding reference frame as shown Fig. 2.3 (b). Denote the common reference frame as DQ reference frame and the angle of the $i^{\text {th }}$ inverter with respect to the DQ-axis is denoted by $\delta_{i}$. Then according to the definition of the terminal voltage angle in equation $(2.2)$, we get

$$
\frac{d \delta_{i}}{d t}=\omega_{i}-\omega_{\mathrm{com}}
$$

When integrated to the microgrid through PCC, the voltage and current of the PCC should be transformed to the common reference frame DQ-axis. Denote $v_{\mathrm{o}}^{i}$ as the terminal voltage, and $v_{\mathrm{b}}^{i}$ as the PCC voltage of the $i^{t h}$ inverter. According to Fig. 2.3 (b), the angle of $v_{\mathrm{o}}^{i}$ with respect to DQ-axis is $\delta_{i}$, and the angle of $v_{\mathrm{b}}^{i}$ is $\theta_{i}+\delta_{i}$.

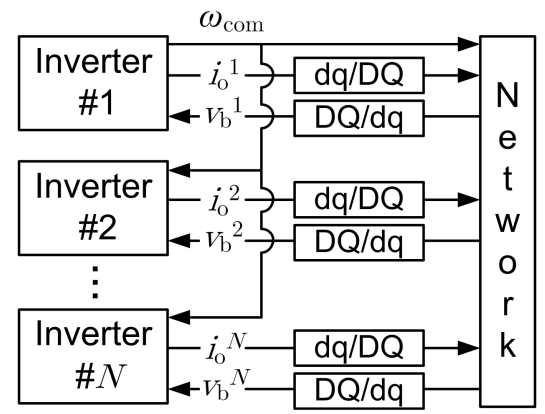

(a)

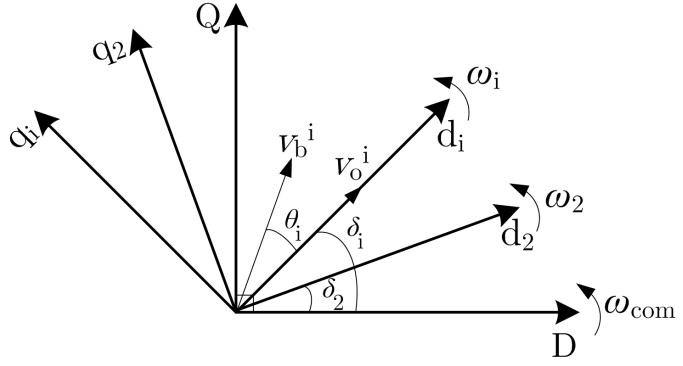

(b)

Figure 2.3: Multiple inverters integrated to microgrid: (a) block diagram; (b) angle reference frames.

Consequently, a microgrid which contains $N$ inverters, the coordinate transformation between the common DQ-axis and the dq-axis for the individual inverters is given by

$$
v_{\mathrm{o}}^{\mathrm{DQ}}=T v_{\mathrm{o}}
$$


where the dq-axis to DQ-axis transformation matrix $T$ is given by

$$
T=\left[\begin{array}{cccc}
e^{\mathrm{j} \delta_{1}} & 0 & \cdots & 0 \\
0 & e^{\mathrm{j} \delta_{2}} & \cdots & 0 \\
\vdots & \vdots & \ddots & \vdots \\
0 & 0 & \cdots & e^{\mathrm{j} \delta_{N}}
\end{array}\right]
$$

A similar coordinate transformation is employed for the inverter output currents. The inverse transformation from DQ-axis to dq-axis is given by $T^{-1}$. Equations (2.2), 2.3), 2.8, 2.9) and 2.10 constitute the ninth-order model of a single inverter.

\subsection{Network Model}

Given the high switching frequency of the inverters, the dynamics of the transmission lines and loads are neglected. Consequently, network interactions are captured by algebraic relations based on Ohm's law and Kirchoff's laws.

Suppose, that in addition to the $N$ inverter buses, the microgrid includes $M$ buses, that may be connected to loads. The microgrid buses are collected in the set $\mathcal{N}:=$ $\{0,1, \ldots, N+M\}$, and distribution lines represented by the set $\mathcal{E}:=\{(m, n)\} \subset \mathcal{N} \times \mathcal{N}$ are modeled as $\pi$-equivalent circuits. The series and shunt admittances of the line $(m$, $n)$ are given by $y_{\mathrm{mn}}=\left(R_{m n}+\mathrm{j} \omega_{\mathrm{com}} L_{\mathrm{mn}}\right)^{-1}$ and $\bar{y}_{\mathrm{mn}}=\mathrm{j} \omega_{\mathrm{com}} C_{\mathrm{mn}}$ respectively, where $R_{m n}, L_{\mathrm{mn}}, C_{\mathrm{mn}}$ and $\omega_{\text {com }}$ denote the line resistance, inductance, shunt capacitance, and the common angular frequency. Let $Y \in \mathbb{C}^{N+M \times N+M}$ denotes the complex admittance matrix of the network, $v, i$ denote the vectors of nodal voltage and nodal current injection ( $i$ and $v$ are both expressed in DQ coordinates). Expressing Kirchoff's circuit laws in matrix-vector form, we can write the network model (including the transmission lines and loads) as

$$
i=Y v \text {. }
$$

where the entries of $Y$ are defined as

$$
[Y]_{\mathrm{m}, \mathrm{n}}:= \begin{cases}\sum_{j \in \mathcal{N}_{\mathrm{m}}}\left(\bar{y}_{\mathrm{mj}}+y_{\mathrm{mj}}\right), & \text { if } m=n \\ -y_{\mathrm{mn}}, & \text { if }(m, n) \in \mathcal{E} \\ 0, & \text { otherwise }\end{cases}
$$


and $\mathcal{N}_{\mathrm{m}}:=\{j \in \mathcal{N}:(m, j) \in \mathcal{E}\}$ denotes the set of buses connected to the $m$ th bus through a distribution line.

For the microgrid of Fig. $2.4(M=3, N=3)$, the admittance matrix is given by

$Y=\left[\begin{array}{cccccc}Z_{14}^{-1} & 0 & 0 & -Z_{14}^{-1} & 0 & 0 \\ 0 & Z_{25}^{-1} & 0 & 0 & -Z_{25}^{-1} & 0 \\ 0 & 0 & Z_{36}^{-1} & 0 & 0 & -Z_{36}^{-1} \\ -Z_{14}^{-1} & 0 & 0 & Z_{14}^{-1}+Z_{45}^{-1}+Z_{1}^{-1} & -Z_{45}^{-1} & 0 \\ 0 & -Z_{25}^{-1} & 0 & -Z_{45}^{-1} & Z_{25}^{-1}+Z_{45}^{-1}+Z_{56}^{-1} & -Z_{56}^{-1} \\ 0 & 0 & -Z_{36}^{-1} & 0 & -Z_{56}^{-1} & Z_{36}^{-1}+Z_{56}^{-1}+Z_{2}^{-1}\end{array}\right]$

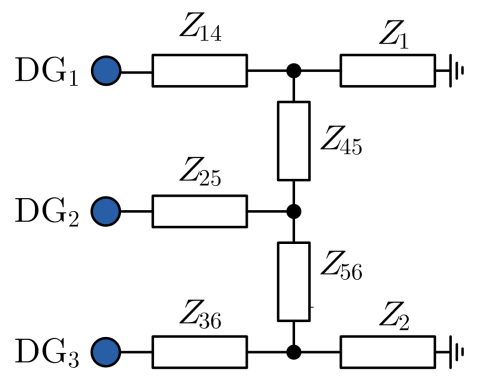

Figure 2.4: Oneline diagram of a sample microgrid with three inverters (inverters are denoted by solid blue dots). 


\section{Chapter 3}

\section{Temporal Model Reduction}

In Chapter 2, we discussed a ninth-order large-signal dynamic model of droop-controlled inverters, which include myriad states from internal control loops and filters [4, 8, 5]. Conceivably, control design, numerical simulations, and stability assessment with such models in islanded microgrids comprising tens of or even hundreds of inverters is computationally expensive and do not offer any analytical insights.

In this Chapter, we propose a model-reduction method based on singular perturbation to obtain reduced-order models for individual inverters. Based on the proposed method, the original ninth-order model includes states of power controller, voltage and current controllers, and inductive filter $\left(x=\left[\delta, S, \phi, \gamma, i_{\mathrm{o}}\right]^{\mathrm{T}}\right)$, which is suitable for detailed studies that capture controller dynamics, is reduced to fifth-, third-, and single-order models.

The reduced order large-signal dynamic models are useful in different contexts. The fifth-order model only considers the angle, power and current dynamics $\left(x=\left[\delta, S, i_{\mathrm{o}}\right]^{\mathrm{T}}\right)$, which can be used to analyze output current and power dynamics; the reduced thirdorder model $\left(x=[\delta, S]^{\mathrm{T}}\right)$ is sufficiently accurate for dynamic simulation and reliability assessment of large microgrids, and the single-order model which has a unique state variable $(x=\delta)$ is suitable for design of secondary-level controllers. 


\subsection{Temporal Model Reduction Based on Singular Pertur- bation Methods}

For dynamical systems which exhibit different dynamic response speeds, fast dynamic variables represent the states which respond faster, and slow dynamic variables represent states that respond slower. The main idea of singular perturbation methods is to reduce model order by eliminating the fast dynamic state variables. Thus, fast dynamic variables are assumed to instantaneously reach its quasi-steady-state solution, which governed by algebraic equations [12, 14, 18, 20, 29].

The dynamic model of a system with multiple time scales can be written in the standard singular perturbation form [12, 13, 30]

$$
\begin{aligned}
\frac{d x}{d t} & =f(x, z, u, t, \varepsilon), \\
\varepsilon \frac{d z}{d t} & =g(x, z, u, t, \varepsilon),
\end{aligned}
$$

where $x \in \mathbb{C}^{n \times 1}$, is the vector that collects the slow dynamic variables, $z \in \mathbb{C}^{m \times 1}$, is the vector that collects the fast dynamic variables, and $u$ is the input, $\varepsilon=\operatorname{diag}\left\{\varepsilon_{1}, \varepsilon_{2}, \cdots, \varepsilon_{m}\right\}$ denotes a diagonal matrix with non-zero entries comprised of the small-perturbation parameters.

From [13, Theorem 11.1], we can reduce the system above to a $n$ th-order model by setting $\varepsilon=0$ and obtaining the the quasi-steady-state solution

$$
z=h(x, u, t)
$$

Substitute $h(x, u, t)$ in $(3.2)$, we get the reduced-order model

$$
\frac{d x}{d t}=f(x, h(x, u, t), u, t, 0),
$$

To accomplish the above steps, we require the Jacobian of $g(\cdot)$, given by

$$
\left.\frac{\partial g(x, z, u, t, \varepsilon)}{\partial z}\right|_{\varepsilon=0}
$$

to be nonsingular and have eigenvalues with negative real parts [13, 30].

To apply the temporal model reduction method proposed in Section 3.1, we need to analyze the different time scales of the droop-controlled inverters. To this end, we will 
use parameters from [4] as a reference.

First, denote $\tau_{\mathrm{S}}, \tau_{\phi}, \tau_{\gamma}$ and $\tau_{\mathrm{f}}$ as the time constants of the power controller, voltage controller, currrent controller and the inductive filter, respectively. The values of the time constants are given by

$$
\begin{aligned}
\tau_{\mathrm{S}} & =\frac{1}{\omega_{\mathrm{c}}}=\frac{1}{31.41}=3.18 \times 10^{-2} \mathbf{s} \\
\tau_{\phi} & =\frac{1}{K_{\mathrm{i}}^{\phi}}=\frac{1}{390}=2.3 \times 10^{-3} \mathbf{s} \\
\tau_{\gamma} & =\frac{1}{K_{\mathrm{i}}^{\gamma}}=\frac{1}{16000}=6.25 \times 10^{-5} \mathbf{s} \\
\tau_{\mathrm{f}} & =\frac{L_{\mathrm{f}}}{R_{\mathrm{f}}}=\frac{1.35 \times 10^{-3}}{0.1}=1.35 \times 10^{-2} \mathbf{s}
\end{aligned}
$$

Notice that the time constants of state variables are quite different from each other, which indicate that droop-controlled inverters have explicit multiple time scales. The current controller responds fastest (with time constant of $6.25 \times 10^{-5} \mathrm{~s}$ ) and the voltage controller dynamics are the second fastest (with time constant of $2.3 \times 10^{-3}$ s). The inductive filter and the power controller react relatively slower (with time constant about $\left.10^{-2} \mathrm{~s}\right)$.

We pick $\varepsilon=\operatorname{diag}\left\{\varepsilon_{1}, \varepsilon_{2}, \varepsilon_{3}, \varepsilon_{4}\right\}=\operatorname{diag}\left\{\frac{1}{K_{\mathrm{i}}^{\phi}}, \frac{1}{K_{\mathrm{i}}^{\gamma}}, \frac{1}{\omega_{\mathrm{c}}}, \frac{L_{\mathrm{f}}}{R_{\mathrm{f}}}\right\}$. In the following sections, we will successively reduce the ninth-order model described in Section 2.1 to a fifth-order model, a third-order model, and finally a single-order model.

\subsection{Reduced Fifth-Order Model}

First, we will reduce the ninth-order model to a fifth-order model by eliminating the dynamical equations for the voltage and current controllers. Notice that the full dynamical model for each inverter, as described by (2.2), (2.3), 2.8), 2.9) and (2.10), includes nine state variables, $\delta, P, Q, \phi_{\mathrm{d}}, \phi_{\mathrm{q}}, \gamma_{\mathrm{d}}, \gamma_{\mathrm{q}}, i_{\mathrm{od}}$ and $i_{\mathrm{oq}}$. For the purpose of analysis, we will find it useful to write (2.6) and (2.7) in the following form

$$
\begin{gathered}
\frac{1}{K_{\mathrm{i}}^{\phi}} \frac{d \phi}{d t}=-\frac{1}{K_{\mathrm{p}}^{\phi}} \phi-\frac{i_{\mathrm{o}}^{\mathrm{ref}}-F i_{\mathrm{o}}}{K_{\mathrm{p}}^{\phi} K_{\mathrm{i}}^{\phi}}, \\
\frac{1}{K_{\mathrm{i}}^{\gamma}} \frac{d \gamma}{d t}=-\frac{1}{K_{\mathrm{p}}^{\gamma}} \gamma-\frac{v_{\mathrm{i}}^{\text {ref }}-\mathrm{j} \omega_{\mathrm{nom}} L_{\mathrm{f}} i_{\mathrm{o}}}{K_{\mathrm{p}}^{\gamma} K_{\mathrm{i}}^{\gamma}},
\end{gathered}
$$


We can rewrite the ninth-order model (as described in equations 2.2 , (2.3), 2.10), (3.6) and (3.7) of a single inverter in the standard singular perturbation form [12, 13, 30].

$$
\begin{gathered}
\frac{d x}{d t}=f(x, z, u, t, \varepsilon), \\
\varepsilon \frac{d z}{d t}=g(x, z, u, t, \varepsilon) .
\end{gathered}
$$

In (3.8) and (3.9),$x=\left[\delta, S, i_{\mathrm{o}}\right]^{\mathrm{T}} \in \mathbb{C}^{3 \times 1}$, is the vector that collects the slow dynamic variables, $z=[\phi, \gamma]^{\mathrm{T}} \in \mathbb{C}^{2 \times 1}$, is the vector that collects the fast dynamic variables, and $u=v_{\mathrm{b}}$ is the input (the PCC bus voltage is adopted as the input). In addition, $\varepsilon=\operatorname{diag}\left\{\varepsilon_{1}, \varepsilon_{2}\right\}=\operatorname{diag}\left\{\frac{1}{K_{\mathrm{i}}^{\phi}}, \frac{1}{K_{\mathrm{i}}^{\gamma}}\right\}$ denotes a diagonal matrix with non-zero entries comprised of the small-perturbation parameters. It is easy to show that the vector fields $f(\cdot)$ and $g(\cdot)$ are given by

$$
\begin{gathered}
f=\left[\begin{array}{c}
\omega_{\text {nom }}-m_{\mathrm{P}} \frac{S+S^{*}}{2}-\omega_{\mathrm{com}} \\
-\omega_{\mathrm{c}} S+\omega_{\mathrm{c}} v_{\mathrm{o}} i_{\mathrm{o}}^{*} \\
-\left(\frac{R_{\mathrm{c}}}{L_{\mathrm{c}}}+\mathrm{j} \omega\right) i_{\mathrm{o}}+\frac{v_{\mathrm{o}}-v_{\mathrm{b}}}{L_{\mathrm{c}}}
\end{array}\right], \\
g=\left[\begin{array}{c}
-\frac{1}{K_{\mathrm{p}}^{\phi}} \phi-\varepsilon_{1} \frac{i_{\mathrm{o}}^{\mathrm{ref}}-F i_{\mathrm{o}}}{K_{\mathrm{p}}^{\phi}} \\
-\frac{1}{K_{\mathrm{p}}^{\gamma}} \gamma-\varepsilon_{2} \frac{v_{\mathrm{i}}^{\mathrm{ref}}-\mathrm{j} \omega_{\mathrm{nom}} L_{\mathrm{f}} i_{\mathrm{o}}}{K_{\mathrm{p}}^{\gamma}}
\end{array}\right],
\end{gathered}
$$

The Jacobian of $g(\cdot)$

$$
\left.\frac{\partial g}{\partial z}\right|_{\varepsilon=0}=\left[\begin{array}{cc}
-\frac{1}{K_{\mathrm{P}}^{\phi}} & 0 \\
0 & -\frac{1}{K_{\mathrm{p}}^{\gamma}}
\end{array}\right] .
$$

Notice that the Jacobian is nonsingular, and has two eigenvalues $-\frac{1}{K_{\mathrm{P}}^{\phi}}$ and $-\frac{1}{K_{\mathrm{P}}^{\gamma}}$, which have negative real parts. Setting $\varepsilon=0$ in $(3.8)$ and $(3.9)$ we obtain

$$
\begin{gathered}
\frac{d x}{d t}=f(x, z, u, t, 0), \\
0=g(x, z, u, t, 0)=\left[\begin{array}{c}
-\frac{1}{K_{\mathrm{p}}^{\phi}} \phi \\
-\frac{1}{K_{\mathrm{p}}^{\gamma}} \gamma
\end{array}\right] .
\end{gathered}
$$


Solving (3.14), we obtain the quasi-steady-state solution for $z$ which is given by

$$
z=h(x, u, t)=\left[\begin{array}{l}
0 \\
0
\end{array}\right] .
$$

This implies $\frac{d \phi}{d t}=0$ and $\frac{d \gamma}{d t}=0$. From $(2.8)$ and $(2.9)$, we conclude

$$
v_{\mathrm{o}}^{\mathrm{ref}}=v_{\mathrm{o}}, \quad i_{\mathrm{o}}^{\mathrm{ref}}=i_{\mathrm{o}} .
$$

Substituting the solution of $z$ in (3.13), we can obtain the following reduced fifth-order model of a single droop-controlled inverter:

$$
\frac{d x}{d t}=f(x, h(x, u, t), u, t, 0)=\left[\begin{array}{c}
\omega_{\mathrm{nom}}-m_{\mathrm{P}} \frac{S+S^{*}}{2}-\omega_{\mathrm{com}} \\
-\omega_{\mathrm{c}} S+\omega_{\mathrm{c}} v_{\mathrm{o}}^{\mathrm{ref}} i_{\mathrm{o}}^{*} \\
-\left(\frac{R_{\mathrm{c}}}{L_{\mathrm{c}}}+\mathrm{j} \omega\right) i_{\mathrm{o}}+\frac{v_{\mathrm{o}}^{\mathrm{ref}}-u}{L_{\mathrm{c}}}
\end{array}\right],
$$

where $x=\left[\delta, S, i_{\mathrm{o}}\right]^{\mathrm{T}} \in \mathbb{C}^{3 \times 1}$ is the vector that collects the slow dynamic variables and $u=v_{\mathrm{b}}$. Notice that we have neglected the dynamic of the fast dynamic variables in the description above.

It is easy to interpret the reduced model of inverter by reviewing the block diagram of a single inverter as shown in Fig. 2.2. From a high-level view, the model reduction procedure eliminated the dynamics of the voltage controller and current controller.

In conclusion, (3.17) describes the fifth-order model where only the angle, power and filter current dynamics are retained. The complete model of the fifth-order model is described in Appendix A Section A.3.

\subsection{Reduced Third-Order Model}

We can further reduce the fifth-order model concluded in Section 3.2 to a third-order and a single-order model step by step. The reduced fifth-order model of (3.17) can be rewritten in the standard singular perturbation form as

$$
\begin{aligned}
& \frac{d x}{d t}=f(x, z, u, t, \varepsilon), \\
& \varepsilon \frac{d z}{d t}=g(x, z, u, t, \varepsilon),
\end{aligned}
$$


In (3.18) and $(3.19), x=[\delta, S]^{\mathrm{T}} \in \mathbb{C}^{2 \times 1}$, is the vector that collects the slow dynamic variables, $z=i_{\mathrm{o}} \in \mathbb{C}^{1 \times 1}$, is the complex variable which captures the dynamics of the output current (fast dynamic variable), and $u=v_{\mathrm{b}}$ is the input (the PCC bus voltage is adopted as the input). In addition, $\varepsilon=\varepsilon_{3}=\frac{L_{\mathrm{c}}}{R_{\mathrm{c}}}$. It is easy to show that the vector fields $f(\cdot)$ and $g(\cdot)$ are given by

$$
\begin{aligned}
& f=\left[\begin{array}{c}
\omega_{\mathrm{nom}}-m_{\mathrm{P}} \frac{S+S^{*}}{2}-\omega_{\mathrm{com}} \\
-\omega_{\mathrm{c}} S+\omega_{\mathrm{c}} v_{\mathrm{o}}^{\mathrm{ref}} i_{\mathrm{o}}^{*}
\end{array}\right], \\
& g=-\left(1+\mathrm{j} \omega \varepsilon_{3}\right) i_{\mathrm{o}}+\frac{v_{\mathrm{o}}^{\mathrm{ref}}-u}{R_{\mathrm{c}}},
\end{aligned}
$$

Similarly to the previous section, first, we calculate the gradient of $g(\cdot)$ which is -1 in this case. It is easy to verify that the gradient satisfies the requirements of singular perturbation model reduction. Then, we set $\varepsilon_{3}=0$ in 3.19 to obtain

$$
z=h(x, u, t)=i_{\mathrm{o}}=\frac{v_{\mathrm{o}}^{\mathrm{ref}}-u}{R_{\mathrm{c}}} .
$$

Substituting $i_{\mathrm{o}}$ in (3.18), and we can obtain the reduced third-order model of a single inverter as follows

$$
\frac{d x}{d t}=f(x, h(x, u, t), u, t, 0)=\left[\begin{array}{c}
\omega_{\mathrm{nom}}-m_{\mathrm{P}} \frac{S+S^{*}}{2}-\omega_{\mathrm{com}} \\
-\omega_{\mathrm{c}} S+\omega_{\mathrm{c}} v_{\mathrm{o}}^{\mathrm{ref}}\left(\frac{v_{\mathrm{o}}^{\mathrm{ref}}-u}{R_{\mathrm{c}}}\right)^{*}
\end{array}\right],
$$

Notice that the quasi-steady-state solution of the fast dynamic variable $i_{\mathrm{o}}=\frac{v_{\mathrm{o}}^{\mathrm{ref}}-u}{R_{\mathrm{c}}}$ can be derived by applying Ohm's law to the physical output R-L filter circuit and neglecting the line inductance. The complete set of equations that describe the third-order model are given in Appendix A Section A.2.

\subsection{Reduced Single-Order Model}

Finally, we reduce the third-order model to a single-order model. At this point, the reduced third-order model of Equation $(3.23)$ can be rewritten in the usual standard singular perturbation form as

$$
\frac{d x}{d t}=f(x, z, u, t, \varepsilon)
$$




$$
\varepsilon \frac{d z}{d t}=g(x, z, u, t, \varepsilon),
$$

In (3.24) and (3.25), $x=\delta$ is the variable that collects the slow dynamic variable (terminal voltage angle), $z=S$ is the complex variable which captures the fast dynamic variables (low-pass filtered apparent power), and $u=v_{\mathrm{b}}$ is the input. In addition, $\varepsilon=\varepsilon_{4}=\frac{1}{\omega_{\mathrm{c}}}$. It is easy to show that $f(\cdot)$ and $g(\cdot)$ are given by

$$
\begin{aligned}
& f=\omega_{\text {nom }}-m_{\mathrm{P}} \frac{S+S^{*}}{2}-\omega_{\mathrm{com}}, \\
& g=-S+v_{\mathrm{o}}^{\mathrm{ref}} i_{\mathrm{o}}^{*}
\end{aligned}
$$

As before, first we calculate the gradient of $g(\cdot)$ which is -1 in this case. It is easy to verify that the gradient satisfies the requirements of singular perturbation model reduction. Then, we set $\varepsilon_{4}=0$ in 3.25 and obtain

$$
S=v_{\mathrm{o}}^{\mathrm{ref}} i_{\mathrm{o}}^{*}=v_{\mathrm{o}}^{\mathrm{ref}}\left(\frac{v_{\mathrm{o}}^{\mathrm{ref}}-u}{R_{\mathrm{c}}}\right)^{*} .
$$

Substituting $S$ in (3.24), we can conclude that the reduced single-order model of a single inverter is given by

$$
\begin{gathered}
\frac{d \delta}{d t}=\omega_{\mathrm{nom}}-m_{\mathrm{P}} \frac{S+S^{*}}{2}-\omega_{\mathrm{com}}, \\
S=v_{\mathrm{o}}^{\mathrm{ref}} i_{\mathrm{o}}^{*}=v_{\mathrm{o}}^{\mathrm{ref}}\left(\frac{v_{\mathrm{o}}^{\mathrm{ref}}-u}{R_{\mathrm{c}}}\right)^{*} .
\end{gathered}
$$

It follows that the quasi-steady-state solution of the fast dynamic variable in the reduced third-order model $S=v_{\mathrm{o}}^{\mathrm{ref}} i_{\mathrm{o}}^{*}$ is the definition of the apparent power when neglecting the dynamics of the power controller.

In this Chapter, we reduced the large-signal dynamic model of a single inverter from the ninth-order to a single-order model step by step based on singular perturbation methods. In the next Chapter, the spatial model reduction of the network based on Kron reduction will be presented. The complete models of the different order models are given in Appendix $\mathrm{A}$. 


\section{Chapter 4}

\section{Spatial Model Reduction}

Kron reduction is a standard tool employed in power networks for applications such as transient stability assessment [21, 22]. Here, Kron reduction is utilized to eliminate internal, non-inverter nodes, and isolate the mutual inverter interactions. This allows us to significantly increase simulation efficiency, and provides more insight into the microgrid dynamics [22].

\subsection{Kron Reduction}

Suppose, that in addition to the $N$ inverter nodes, the microgrid includes $M$ nodes, that may be connected to loads. Let $Y \in \mathbb{C}^{N+M \times N+M}$ denotes the complex admittance matrix of the network, $i$ denote the vector of nodal current injections, and let $v$ denote the vector of nodal voltages ( $i$ and $v$ are both expressed in $D Q$ coordinates corresponding to the reference inverter). Expressing Kirchoff's circuit laws in matrix-vector form, we can write

$$
i=Y v .
$$

Let $v_{\alpha} \in \mathbb{C}^{N \times 1}\left(v_{\beta} \in \mathbb{C}^{M \times 1}\right)$ denote the vector of voltages of the nodes connected (not connected, respectively) to inverters, and let $i_{\alpha} \in \mathbb{C}^{N \times 1}\left(i_{\beta} \in \mathbb{C}^{M \times 1}\right)$ denote the vector of current injections at the nodes connected (not connected, respectively) to the 
inverters. Then 4.1 can be expressed as

$$
\left[\begin{array}{c}
i_{\alpha} \\
i_{\beta}
\end{array}\right]=\left[\begin{array}{cc}
Y_{\alpha \alpha} & Y_{\alpha \beta} \\
Y_{\alpha \beta}^{\mathrm{T}} & Y_{\beta \beta}
\end{array}\right]\left[\begin{array}{l}
v_{\alpha} \\
v_{\beta}
\end{array}\right] .
$$

Since the loads in the microgrid are constant impedances or currents, Kron reduction allows us to eliminate $v_{\beta}$, to obtain

$$
i_{\alpha}=\left(Y_{\alpha \alpha}-Y_{\alpha \beta} Y_{\beta \beta}^{-1} Y_{\alpha \beta}^{\mathrm{T}}\right) v_{\alpha}+Y_{\alpha \beta} Y_{\beta \beta}^{-1} i_{\beta}=Y_{\mathrm{Kron}} v_{\alpha}+Y_{\alpha \beta} Y_{\beta \beta}^{-1} i_{\beta}
$$

Basically, (4.3) means the inverter current injections are related to terminal voltages through the admittance matrix of Kron-reduced network $\left(Y_{\mathrm{Kron}}=Y_{\alpha \alpha}-Y_{\alpha \beta} Y_{\beta \beta}^{-1} Y_{\alpha \beta}^{\mathrm{T}}\right)$, and the current loads are mapped to individual inverters through $Y_{\alpha \beta} Y_{\beta \beta}^{-1}$. Furthermore, the absence of $v_{\beta}$ implies that only the mutual inverter interactions between inverters are retained. And, $Y_{\text {Kron }}$ captures equivalent local loads and equivalent series impedance that link inverters.

\subsection{Input of Inverter Dynamical Models}

Assume there are $N$ inverter nodes and $M$ non-inverter nodes in the microgrid. Without loss of generality, assume the first $N$ nodes are connected with inverters while others may be connected to loads. For simplicity of discussion, here we assume there are only impedance loads in the network, then equation (4.1) will be

$$
i_{\alpha}=Y_{\mathrm{kr}} v_{\alpha}=\left(Y_{\alpha \alpha}-Y_{\alpha \beta} Y_{\beta \beta}^{-1} Y_{\alpha \beta}^{\mathrm{T}}\right) v_{\alpha} .
$$

In the dynamic simulation of microgrids, we need to solve two parts (the differentialalgebraic model for the inverters, and the algebraic equations of the network) separately within one iteration. First we solve for bus voltage 1

$$
v_{\alpha}=Y_{\mathrm{Kron}}^{-1} i_{\alpha}
$$

where

$$
i_{\alpha}=T i_{\mathrm{o}}, \quad v_{\alpha}=T v_{\mathrm{b}}
$$

\footnotetext{
1 Generally, the equivalent loads of shunt impedance connected to inverter buses are non-zero, $Y_{\text {Kron }}$ is invertible.
} 
Then, we transform the PCC bus voltage from DQ-axis to the dq-axis for individual inverters by $v_{\mathrm{b}}^{i}=e^{-\mathrm{j} \delta_{i}} v_{\alpha}^{i}$. Written in matrix form, the input of the inverter differential equations is given by

$$
u=v_{\mathrm{b}}=T^{-1} Y_{\mathrm{kr}}^{-1} T i_{\mathrm{o}} .
$$

where the dq-axis to DQ-axis transformation matrix $T$ is defined in equation (2.13). Then, set $v_{b}$ as the input of the differential equations of the inverters and solve the state variables (shown in Chapter 3 ).

\subsection{Software Package Developed in MATLAB}

In Chapters 3 and 4, we obtained reduced-order models of droop-controlled islanded inverters. To test the spatio-temporal reduced order models, a software package including the original thirteenth-order model from [4, 8], the ninth-order and the reduced fifthand third-order models are implemented in MATLAB ${ }^{2}$

The developed software package can create selected order of large-signal dynamic model of inverter-based islanded microgrid with any number of inverters. To make the numerical study results comparable, the same MATLAB functions are used to solve the DAE equations. The flow chart of coding the dynamical system model of different orders (13, $9,5,3)$ in MATLAB is shown in Figure 4.1. The software package in MATLAB consists four functionality blocks:

(1) Input block: read in inverter data, network parameters and load steps information.

(2) Admittance matrix block: which creates $Y$ matrix, and implements Kron reduction (obtains $Y_{\text {Kron }}$ ) for impedance loads and current loads.

(3) Inverter model block: create selected order large-signal dynamic model of microgrids based on input data and calculated admittance matrix.

(4) Output block: output the chosen simulation results, including state variables, power losses, maximum voltage deviations, dq components and abc components of output currents and terminal voltages.

\footnotetext{
${ }^{2}$ A coupling capacitor paralleled to the inductive filter in thirteenth-order model is neglected in the ninth-order model, because the capacitor is really small and it has high natural frequency which is much faster than the dynamics of other electrical variables, such as inductor current, terminal voltage and apparent power.
} 


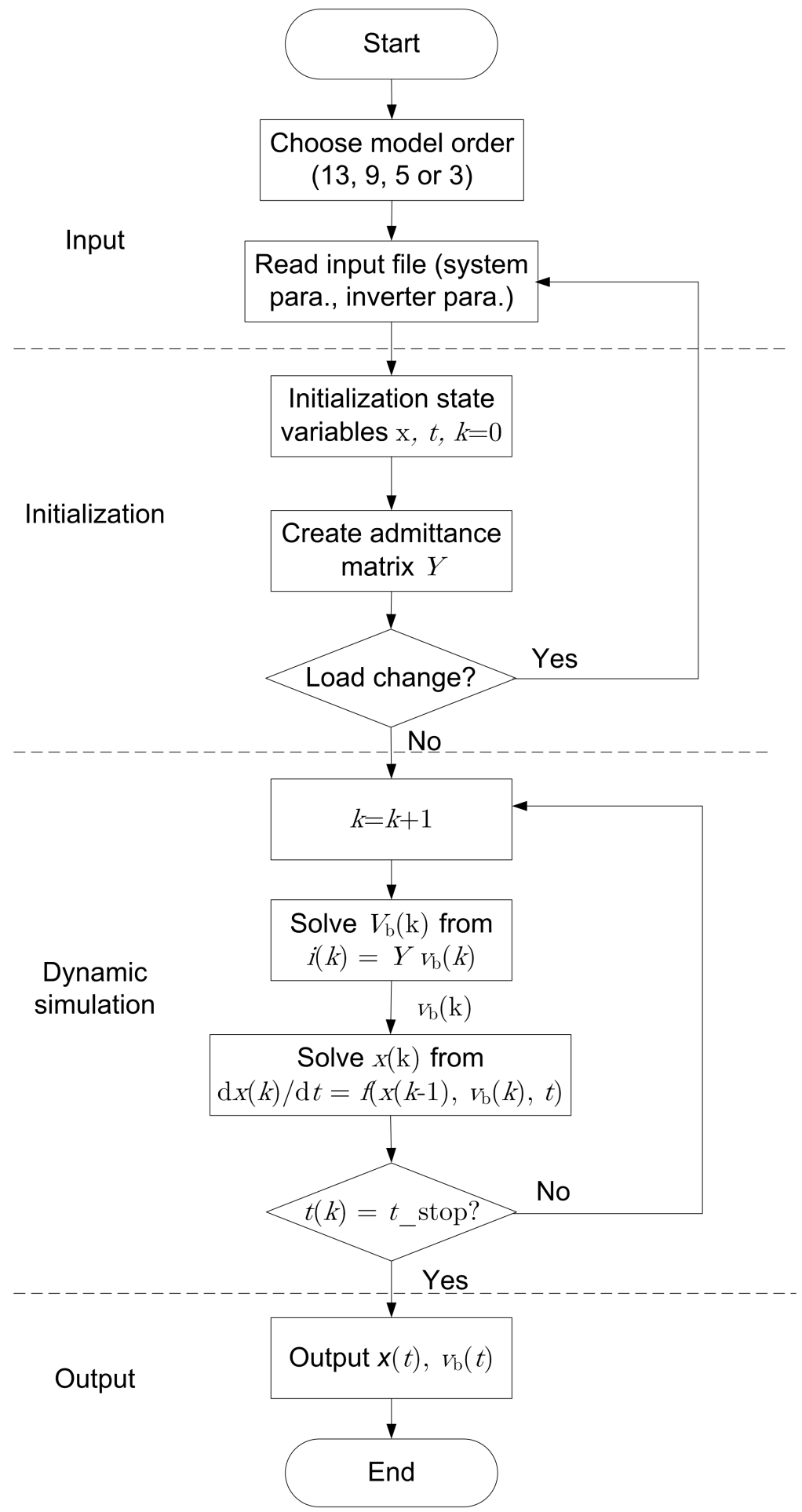

Figure 4.1: Flow chart of dynamic simulations in MATLAB 


\section{Chapter 5}

\section{Numerical Studies}

Based on the MATLAB software package discussed in Section 4.3, two numerical cases are studied in this Chapter. Case 1 is a six-bus microgrid with three inverters and designed at 220V (per-phase RMS); Case 2 is a modified IEEE 37-bus system with seven inverters and designed at $4.8 \mathrm{kV}$ (per-phase RMS). Simulation results and analysis are as follows.

\subsection{Case 1: Six-Bus Microgrid}

Figure 5.1(a) shows a six-bus microgrid with three inverters $(N=3, M=3)$ [4]. The droop-controlled inverters are designed at $220 \mathrm{~V}$ (per phase RMS) and $f=50 \mathrm{~Hz}$. Two loads $Z_{1}$ and $Z_{2}$ are connected to buses 1 and 3 .

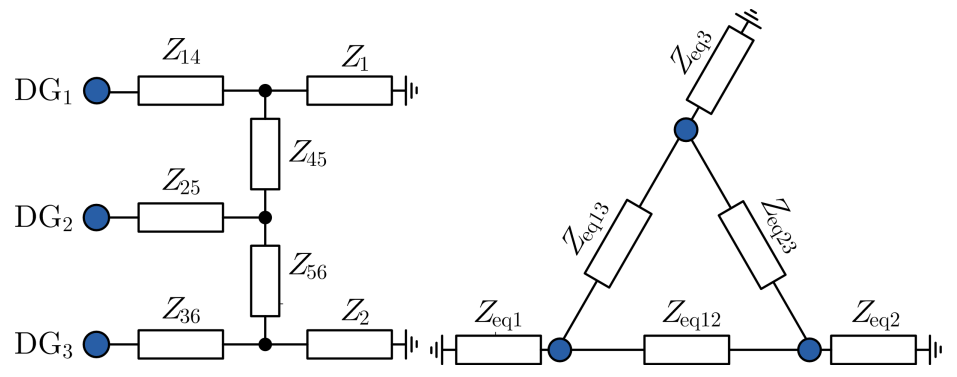

(a)

(b)

Figure 5.1: Oneline diagram of microgrid with three inverters: (a) Original network; (b) Kronreduced network (inverters are depicted with blue dots). 
Assume that the three inverters are equally rated. Thus, we use the same droop coefficients for the frequency and voltage controllers. Furthermore, for the initial studies, we assume the loads are resistive with per-phase parameters listed in Table 5.1 , and current loads are disregarded $\left(i_{\beta}=0\right)$. The Kron-reduced network is shown in Figure 5.1(b).

Table 5.1: Network parameters of Case 1

\begin{tabular}{cccc}
\hline Parameter & Value $(\Omega)$ & Parameter & Value $(\Omega)$ \\
\hline$Z_{14}$ & $0.03+\mathrm{j} 0.11$ & $Z_{25}$ & $0.03+\mathrm{j} 0.11$ \\
$Z_{36}$ & $0.03+\mathrm{j} 0.11$ & $Z_{45}$ & $0.23+\mathrm{j} 0.1$ \\
$Z_{56}$ & $0.35+\mathrm{j} 0.58$ & $Z_{1}$ & 25 \\
$Z_{2}$ & 20 & & \\
\hline
\end{tabular}

For this Kron-reduced network, we will compare the different reduced-order models described next.

\subsubsection{Responses of Fast and Slow Dynamic Variables}

First, to analyze the different time scales of the system state variables, a complete ninthorder model of the test system is simulated (see section A.4 in Chapter A). By running the simulation from start up, the initial conditions of the system for load steps can be obtained. When $t=0.1$ s, a load step signal (change $Z_{1}$ from $25 \Omega$ to $20 \Omega$ ) is applied to the model. The step responses of the fast and slow state variables are shown in Fig. $5.2{ }^{1}$. From the figures, notice that the transients of the fast variables $\phi_{d}, \phi_{q}, \gamma_{d}, \gamma_{q}$ are much faster than the slow variables $\delta, P, Q$.

\footnotetext{
1 In these plots, different colors correspond to different inverters: inverter 1 (dash-dot black line), inverter 2 (solid magenta line) and inverter 3 (dashed blue line)
} 

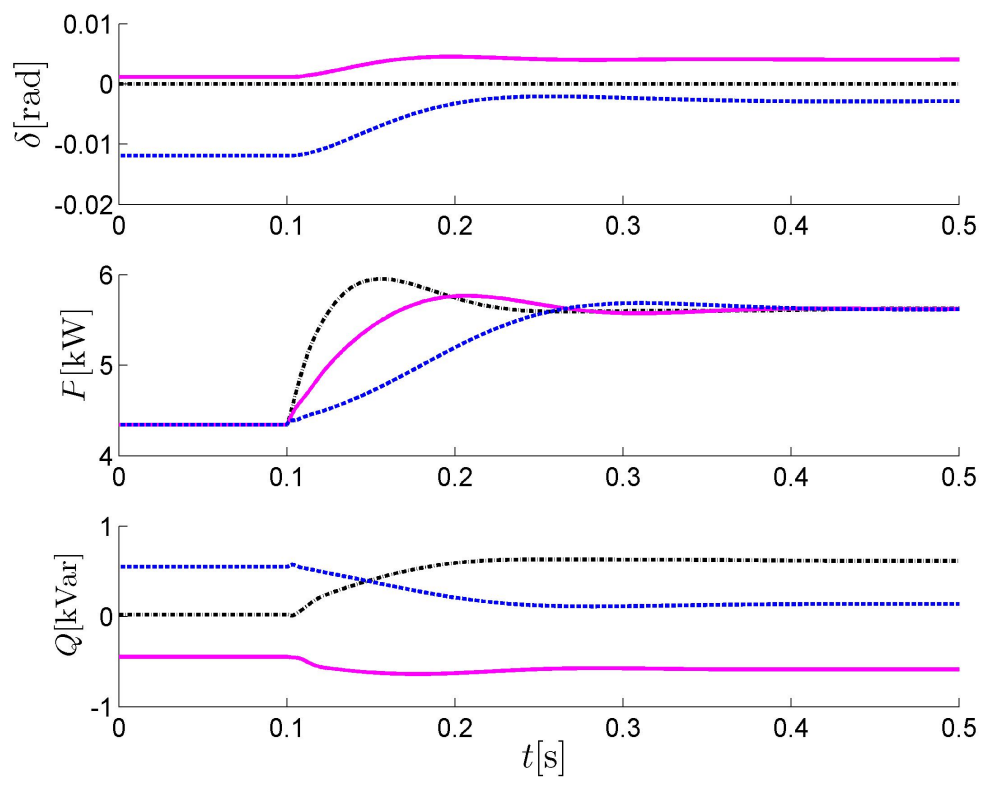

(a)
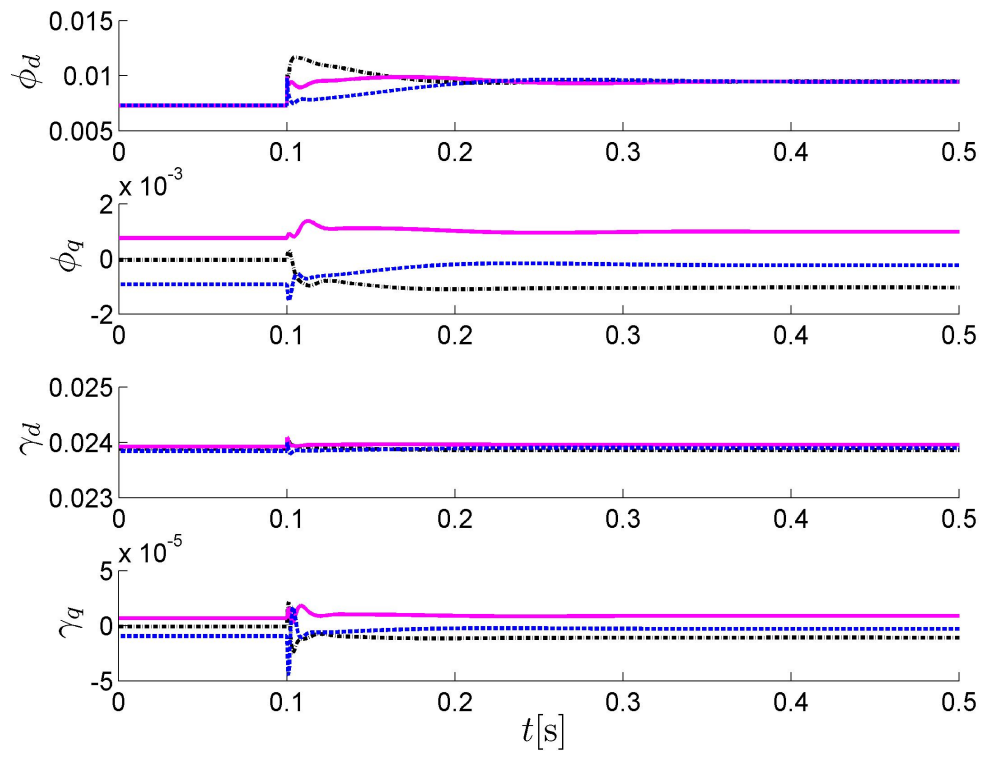

(b)

Figure 5.2: Comparison of slow and fast dynamic variables. (a) Responses of the slow variables $\delta, P, Q$; (b) Responses of the fast variables $\phi_{d}, \phi_{q}, \gamma_{d}, \gamma_{q}$ 


\subsubsection{Original Model Compared to Reduced Models}

To test the different reduced-order models, we first ran the simulation from start up to check if the terminal voltage angles, active power and reactive powers are stable. The simulation results are shown in Fig. 5.3-5.6.

Then we applied a load step signal (change $Z_{1}$ from $25 \Omega$ to $20 \Omega$ ) to the model at $t=0.1 \mathrm{~s}$. The simulation results are shown from Fig. 5.7- $5.10^{2}$

Figures 5.3 - 5.6 show that the transients of terminal voltage angle, active power and reactive power of the spatio-temporal reduced-order models are very close to the fullorder model which verifies that the reduced-order models accurately capture relevant dynamics of interest.

Furthermore, the step responses of $\delta, P$ and $Q$ of reduced order models match well with the thirteenth- and ninth-order model. From Fig. 5.8, three inverters increased the active power from $4.343 \mathrm{~kW}$ to $5.626 \mathrm{~kW}$ to share the load change. Since the three inverters are equally rated and controlled with the same droop coefficients, the active power increments are the same in steady state. However, the load change happens in node 1 which is closest to the first inverter and furthest to the third inverter. It is therefore reasonable that the first inverter responded fastest and the third responded slowest. Besides, since the angle of the first inverter is set to be the common reference angle, the relative angle $\delta_{1}$ is zero which is not shown in the figures.

\footnotetext{
2 In these plots, colors and line styles are defined as thirteenth-order ${ }^{3}$ (dashed black line), ninthorder (solid red line), fifth-order (dotted green line) and third-order (solid blue line).
} 


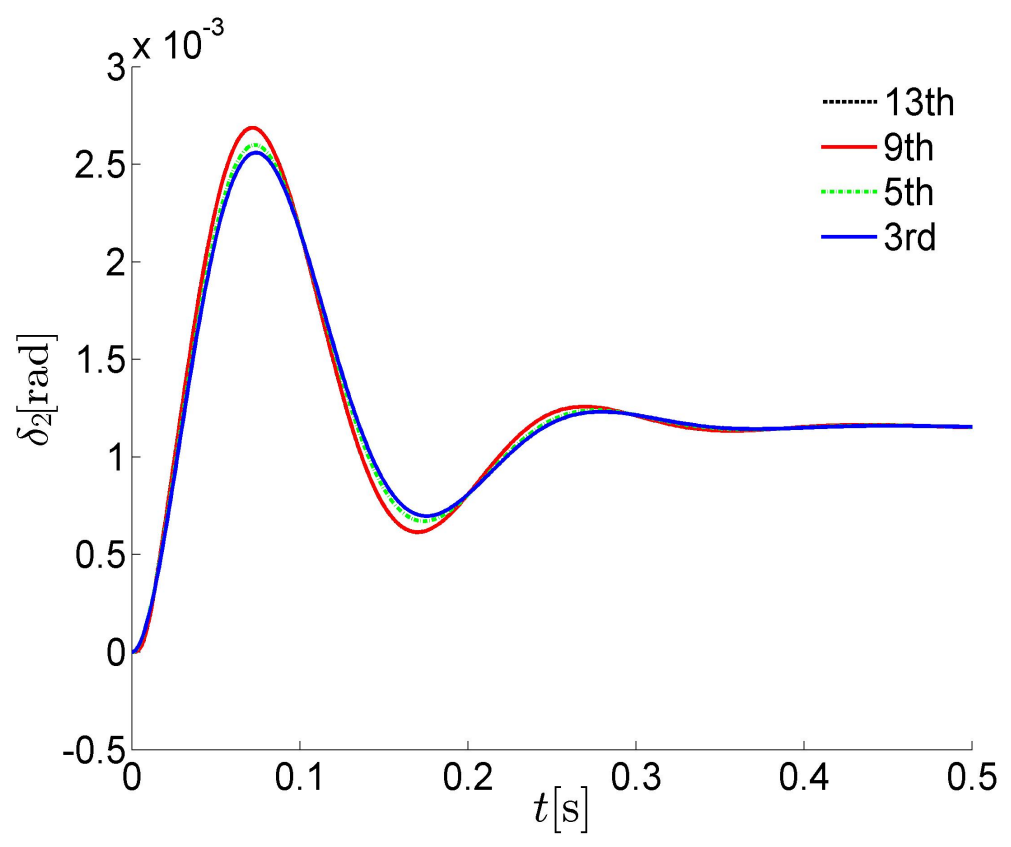

(a)

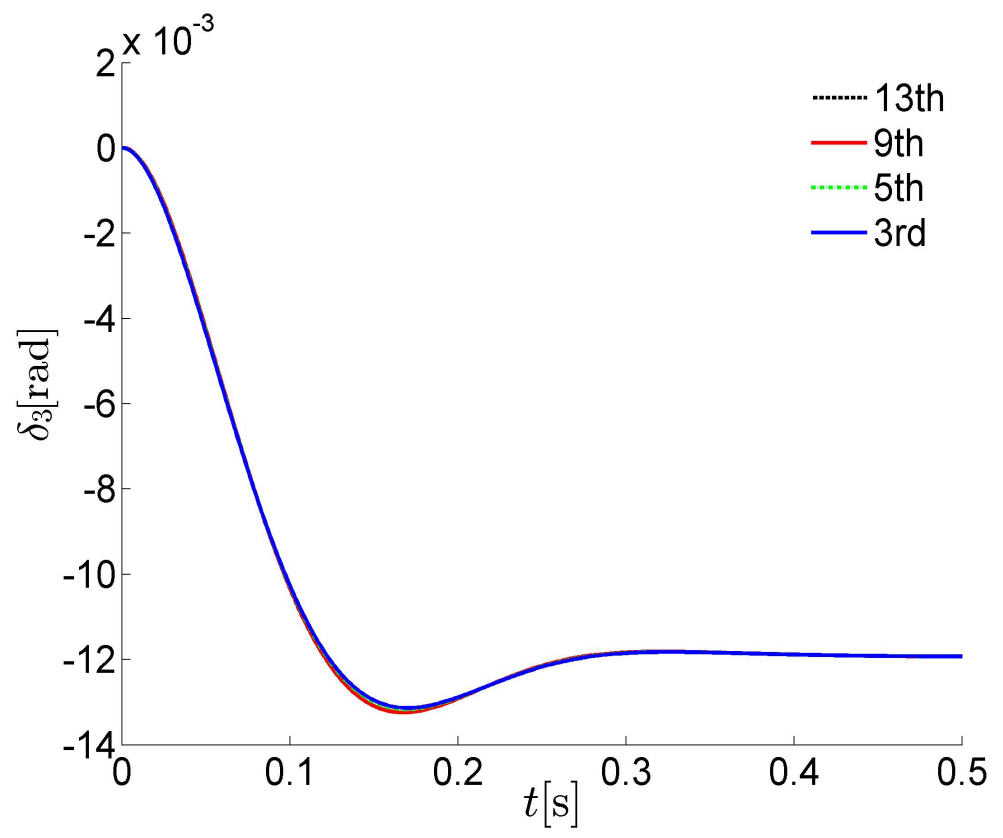

(b)

Figure 5.3: Terminal voltage angle transients from startup comparing the original 13thorder and the spatio-temporal reduced models: (a) $\delta_{2} ;(\mathrm{b}) \delta_{3}$. 


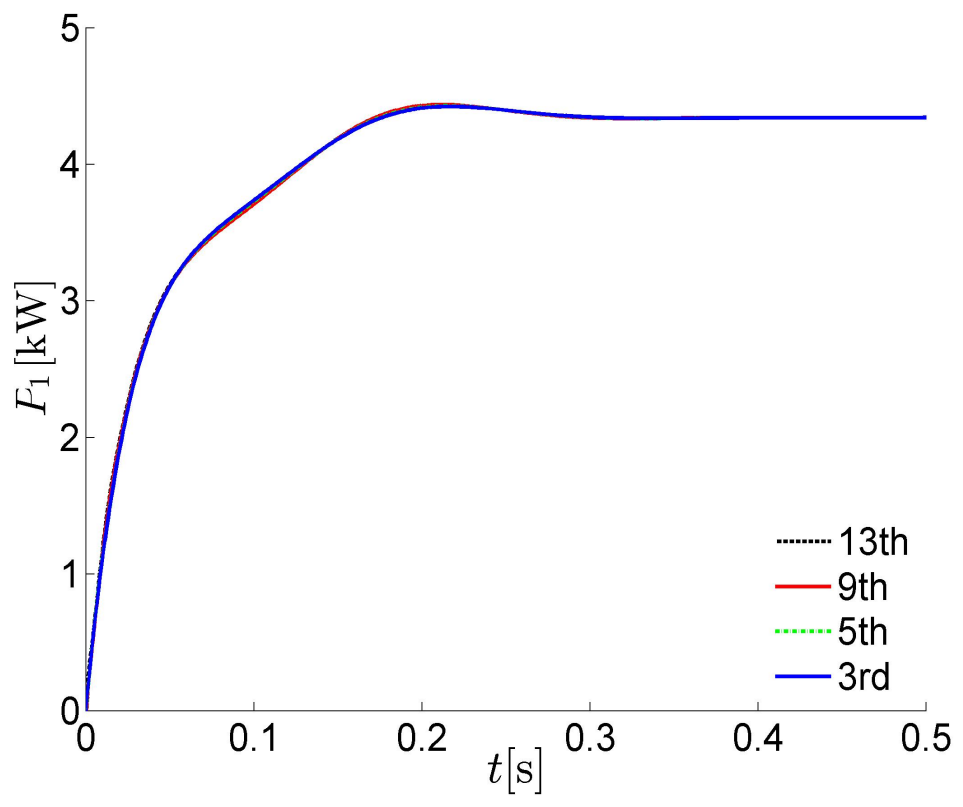

(a)

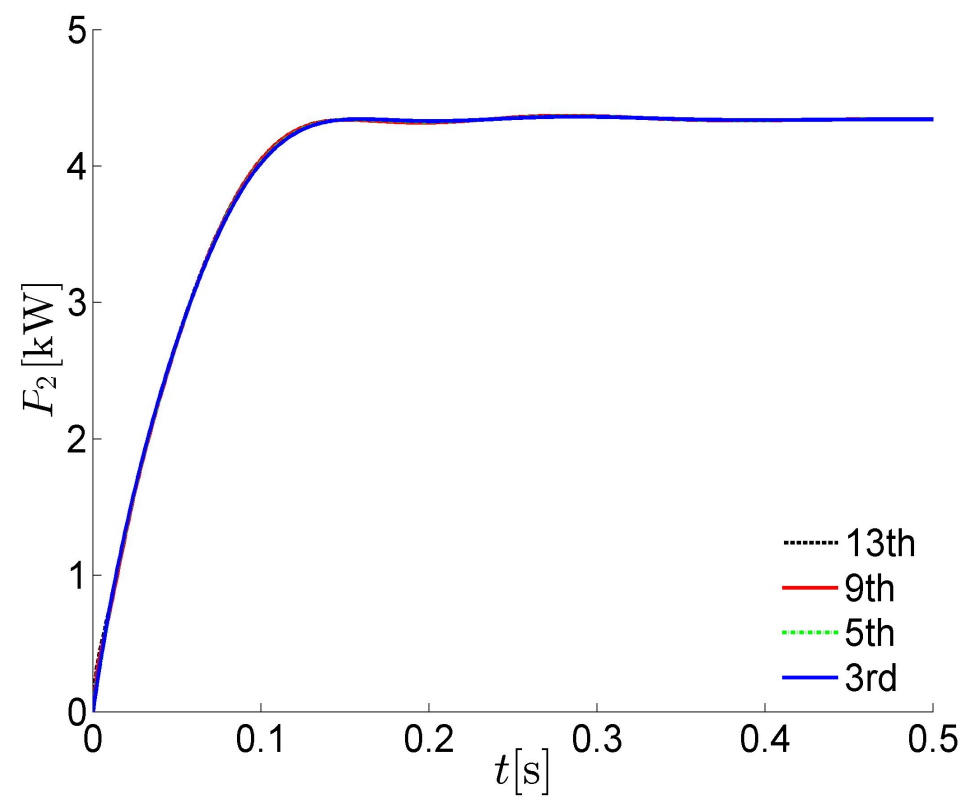

(b)

Figure 5.4: Active power transients from startup comparing the original 13th-order and the spatio-temporal reduced models: (a) $P_{1}$; (b) $P_{2}$. 


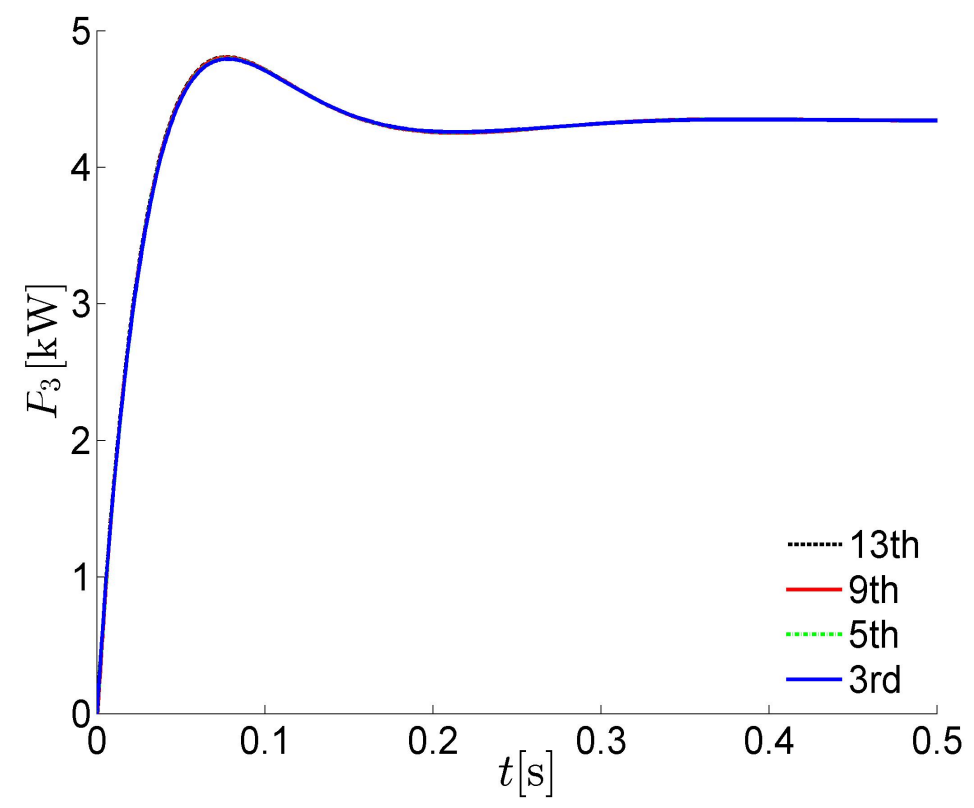

(a)

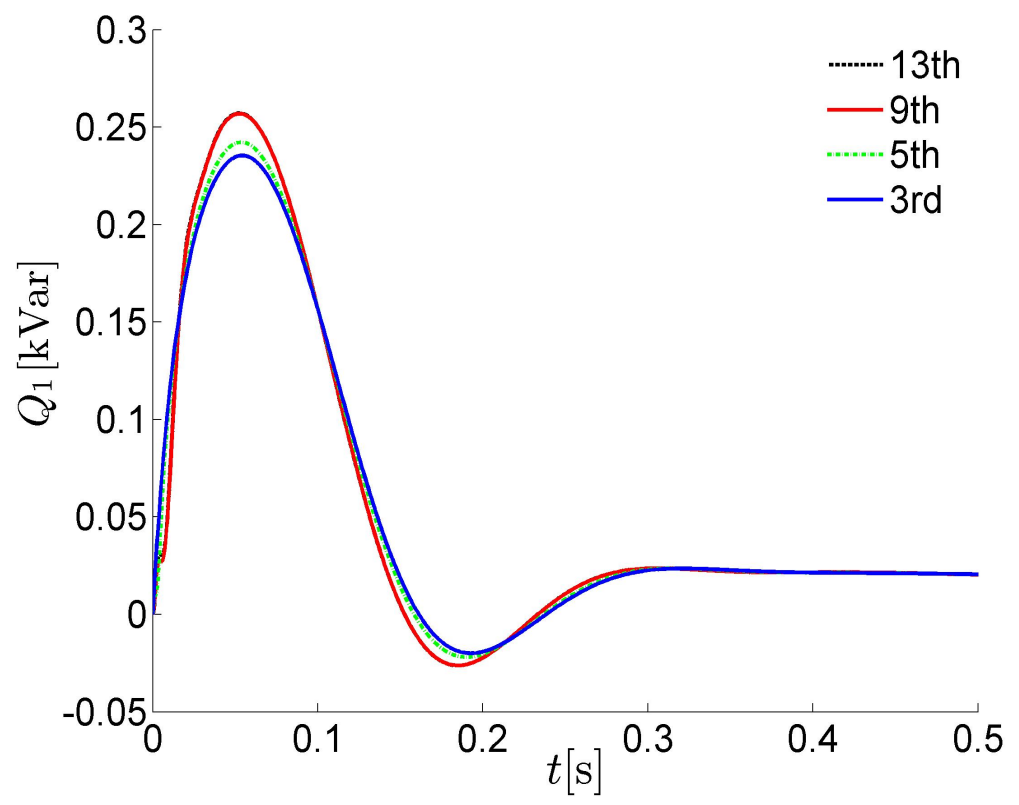

(b)

Figure 5.5: Active and Reactive power transients from startup comparing the original 13th-order and the spatio-temporal reduced models: (a) $P_{3}$; (b) $Q_{1}$. 


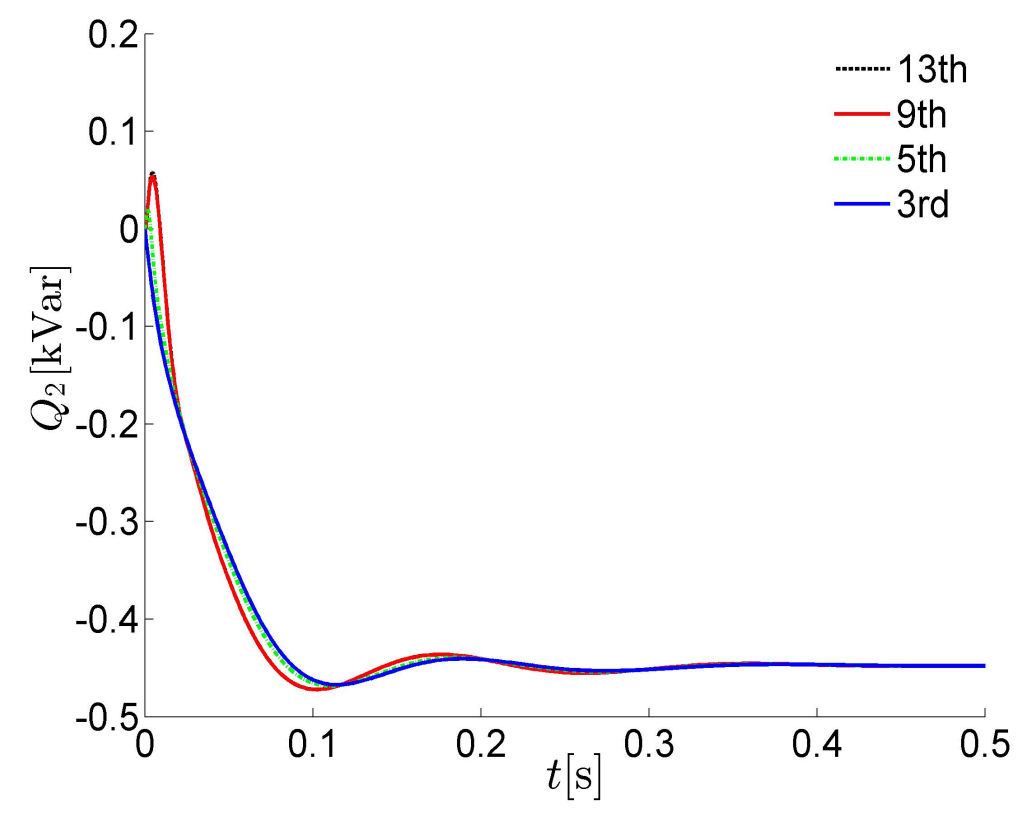

(a)

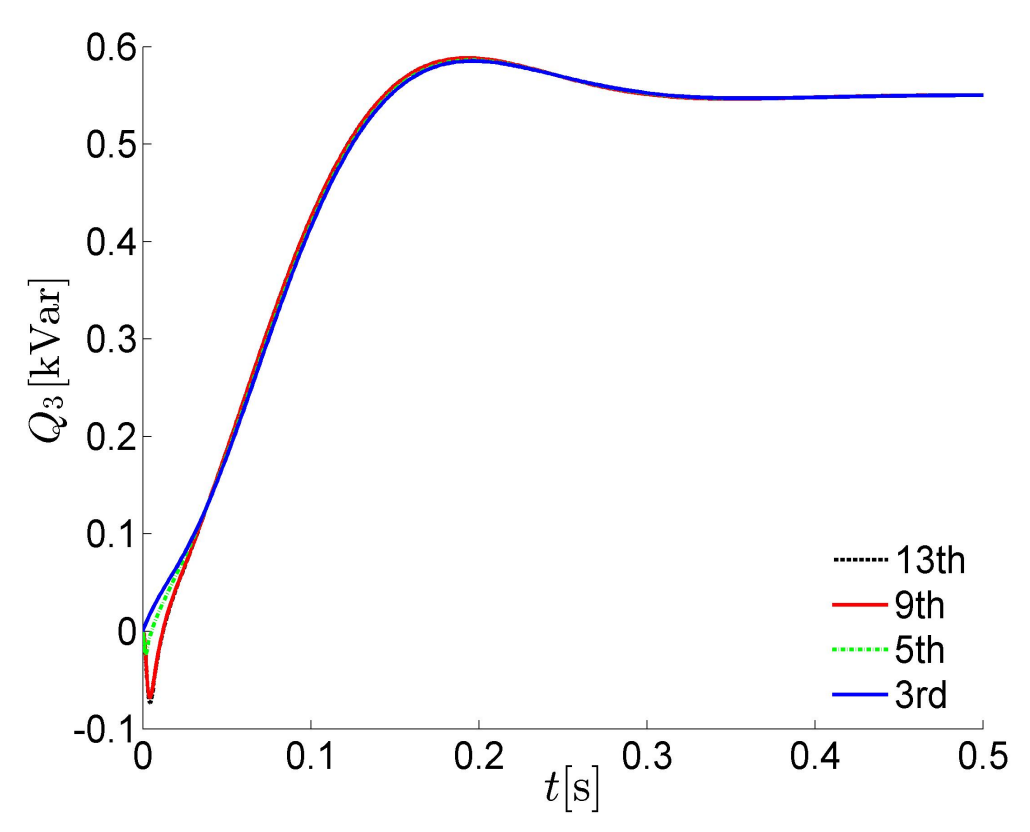

(b)

Figure 5.6: Reactive power transients from startup comparing the original 13th-order and the spatio-temporal reduced models: (a) $Q_{2} ;(\mathrm{b}) Q_{3}$. 


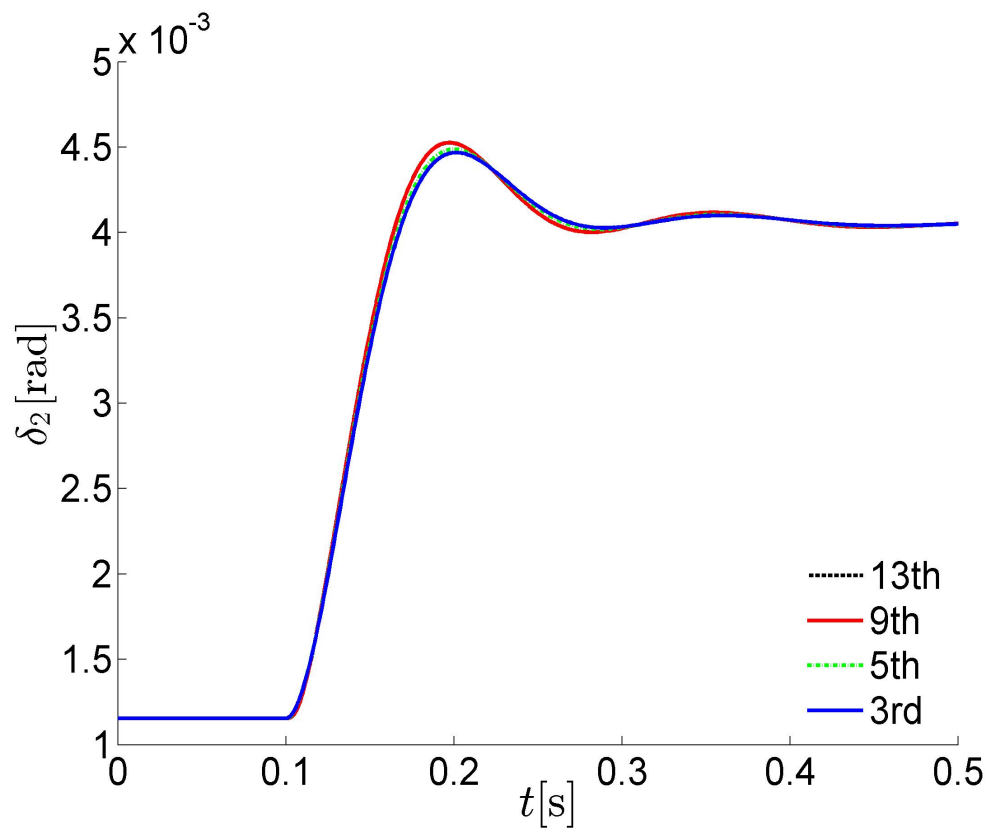

(a)

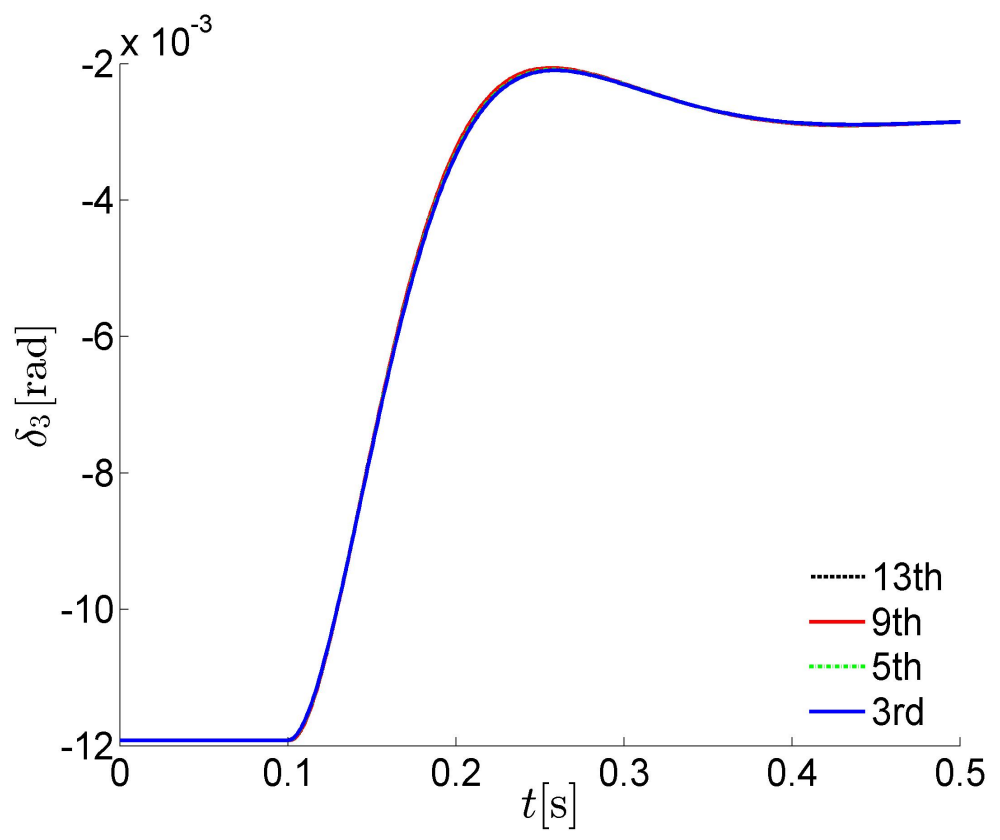

(b)

Figure 5.7: Terminal voltage angle transients from startup comparing the original 13thorder and the spatio-temporal reduced models: (a) $\delta_{2} ;(\mathrm{b}) \delta_{3}$. 


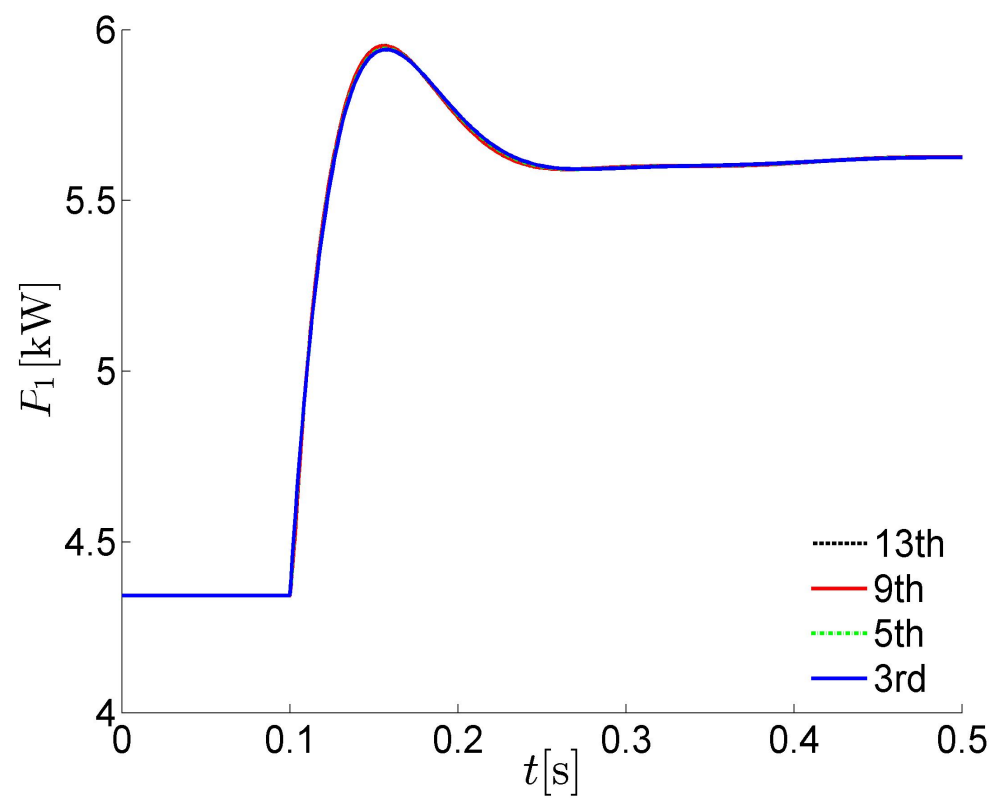

(a)

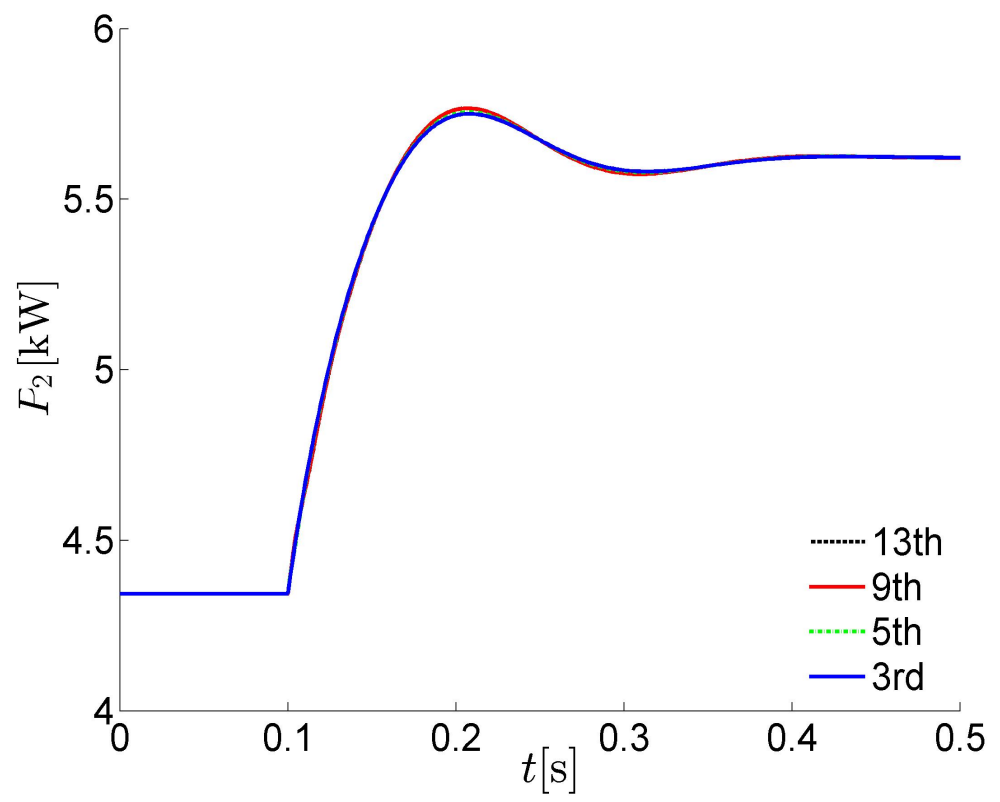

(b)

Figure 5.8: Active power transients from startup comparing the original 13th-order and the spatio-temporal reduced models: (a) $P_{1}$; (b) $P_{2}$. 


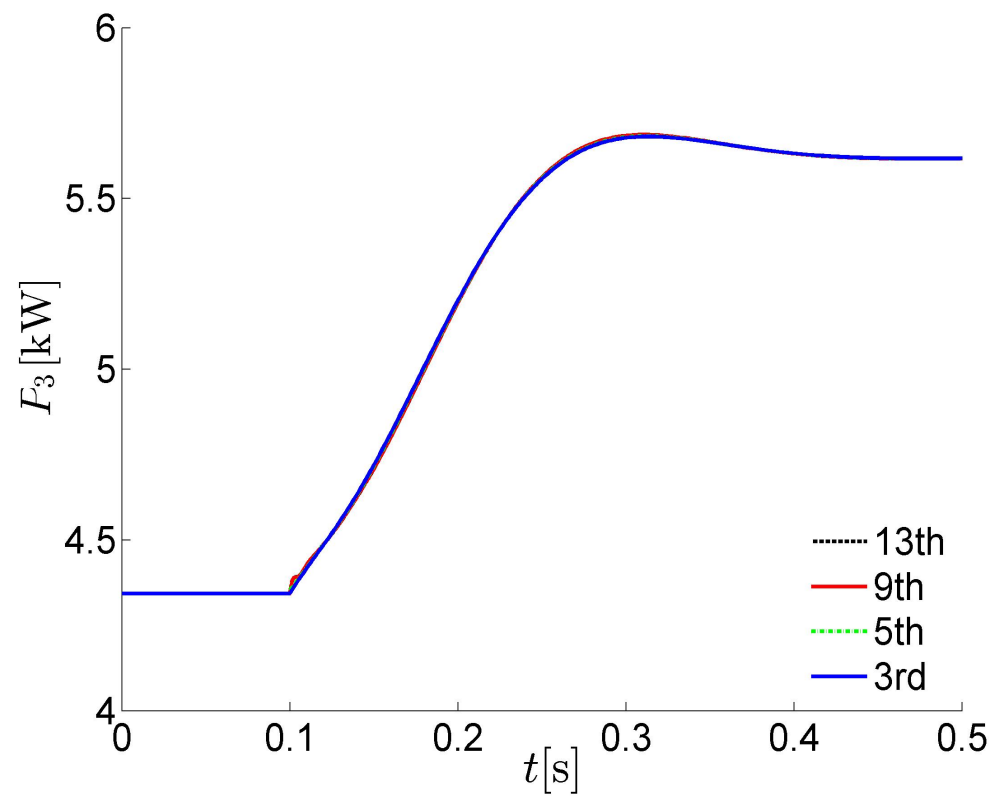

(a)

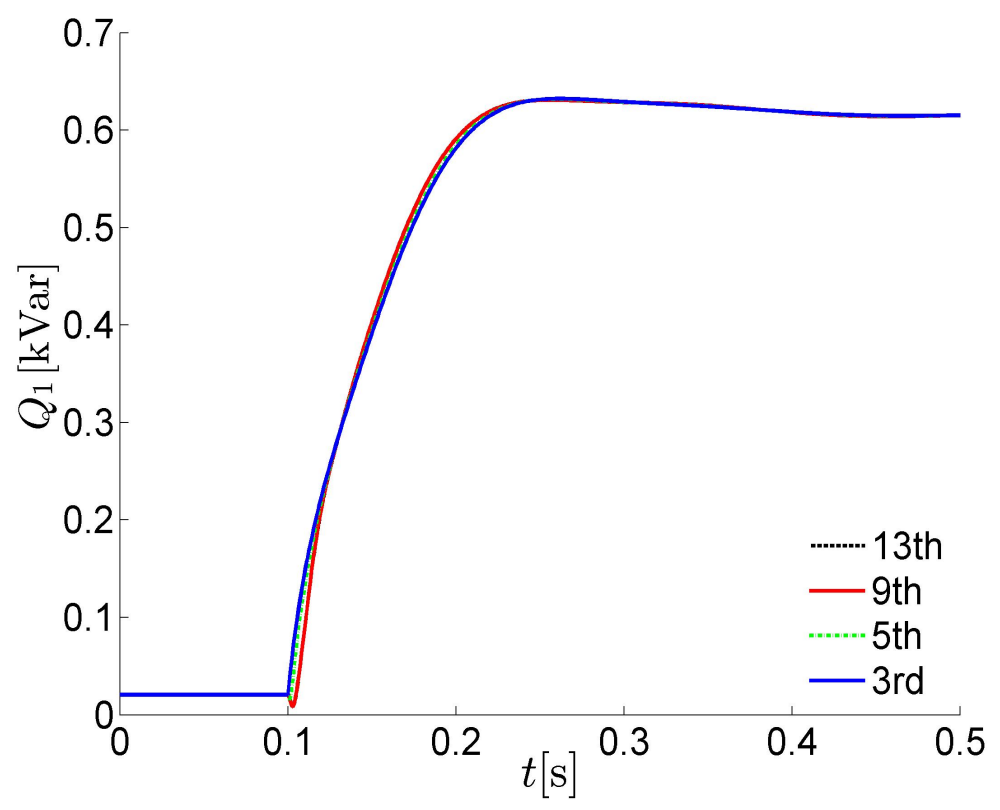

(b)

Figure 5.9: Active and Reactive power transients from startup comparing the original 13th-order and the spatio-temporal reduced models: (a) $P_{3}$; (b) $Q_{1}$. 


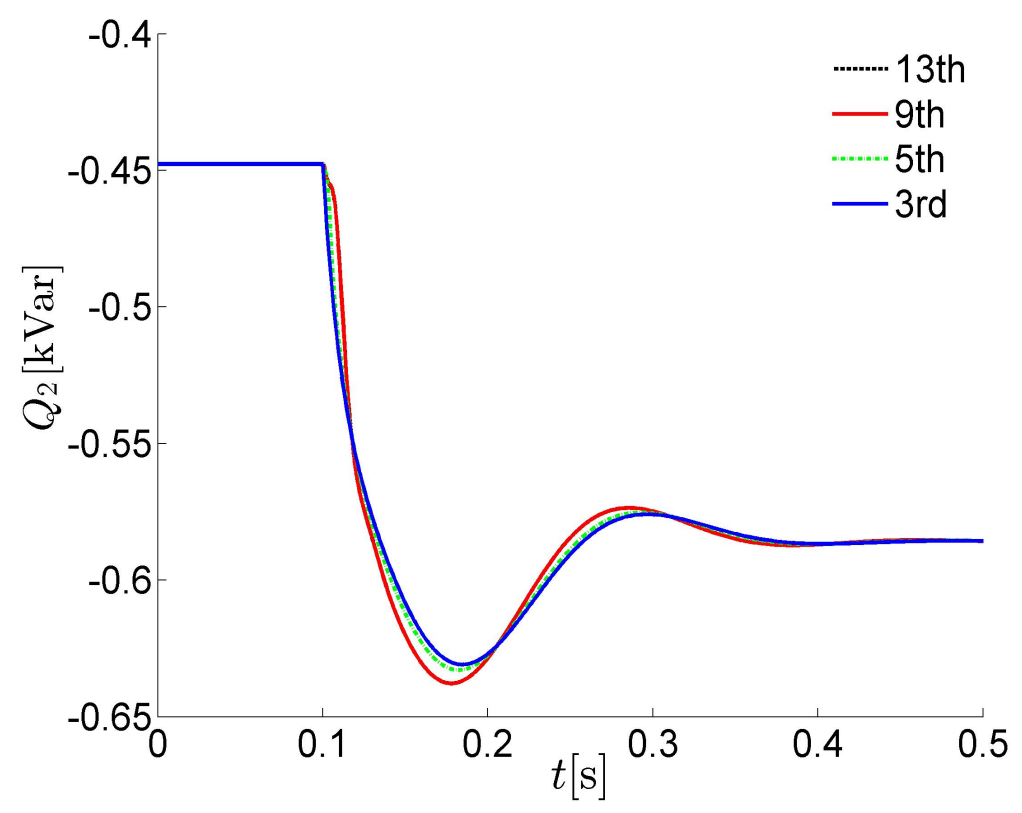

(a)

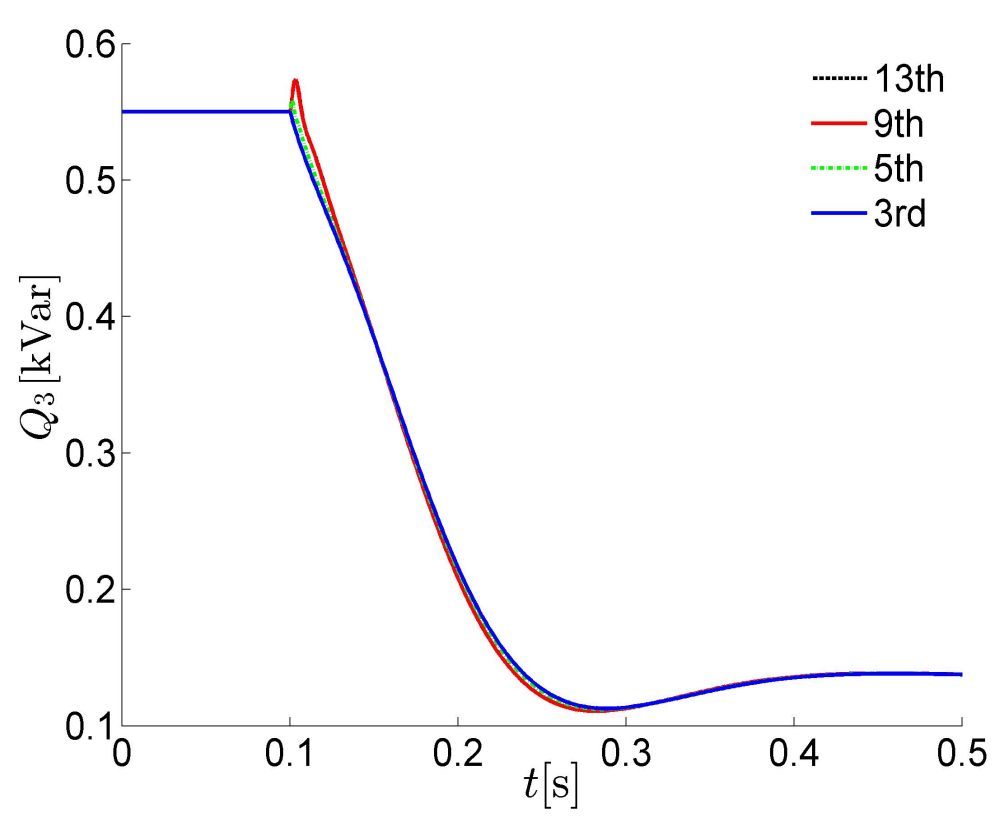

(b)

Figure 5.10: Reactive power transients from startup comparing the original 13th-order and the spatio-temporal reduced models: (a) $Q_{2} ;(\mathrm{b}) Q_{3}$. 


\subsection{Case 2: IEEE 37-Bus Microgrid}

In the previous subsection, a small islanded microgrid was studied. In order to further test the spatio-temporal reduced model, a simulation case study is performed for the IEEE 37-bus system.

To formulate a microgrid, the standard IEEE 37-bus system was modified by adding inverters at certain buses in the circuit as shown in Fig. 5.11. The test system is $4.8 \mathrm{kV}$ (per phase RMS) and $f=50 \mathrm{~Hz}$, thus in the model $v_{\text {nom }}=4.8 \sqrt{3} \mathrm{kV}$. Seven inverters are added at buses 718, 724, 729, 731, 736, 741 and 742. Loads are modeled as impedance and current sources in the system. The system parameters are collected in Table B.2 Table B.4 in the Appendix B [31. The droop coefficients of power droop control and voltage droop control are picked to be $m_{\mathrm{P}}=4.631 e^{-7}$ and $n_{\mathrm{Q}}=5.61 e^{-4}$ for all inverters. The other inverter parameters are the same as the six-bus system in Table B.1 of Appendix B.

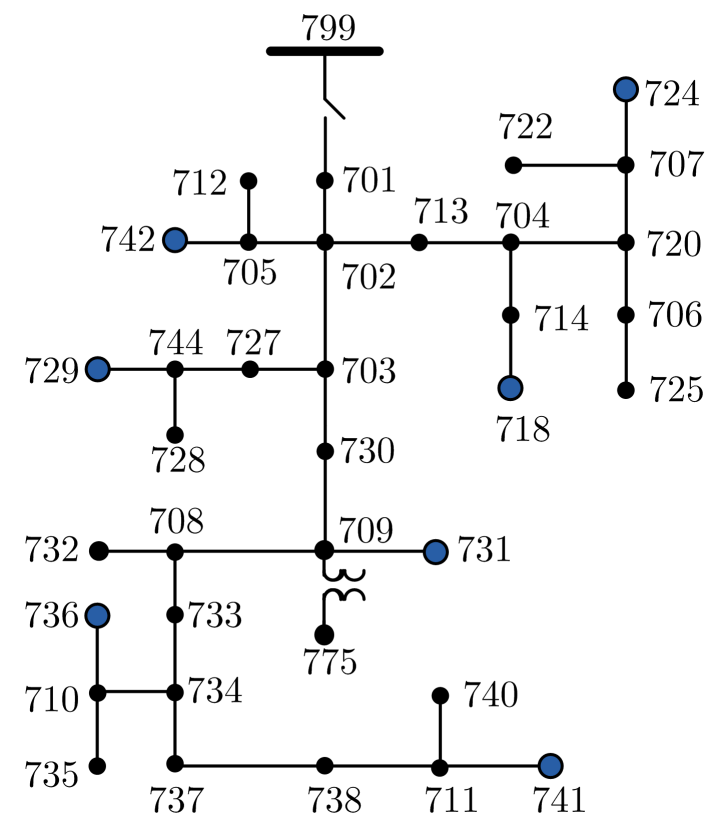

Figure 5.11: One-line diagram of modified IEEE 37-bus test system (inverters are represented by blue dots). 


\subsubsection{Original Model Compared to Reduced Models}

To test the dynamic behavior of the ninth-order model, a load step (change the resistance from $87.7714 \Omega$ to $67.7714 \Omega$ ) is applied to the load connected to bus 701 at $t=$ $0.1 \mathrm{~s}$. The step responses of the terminal voltage angle, active power and reactive power of the seven inverters are shown in Figs. $5.12-5.14$

Furthermore, the simulation results by using different reduced-order models are compared. Since the load change is at bus 701, the electrically closest four inverters (connected to buses 718, 724, 729 and 742) are focused on. The simulation results are shown in Figs. $5.15-5.195$

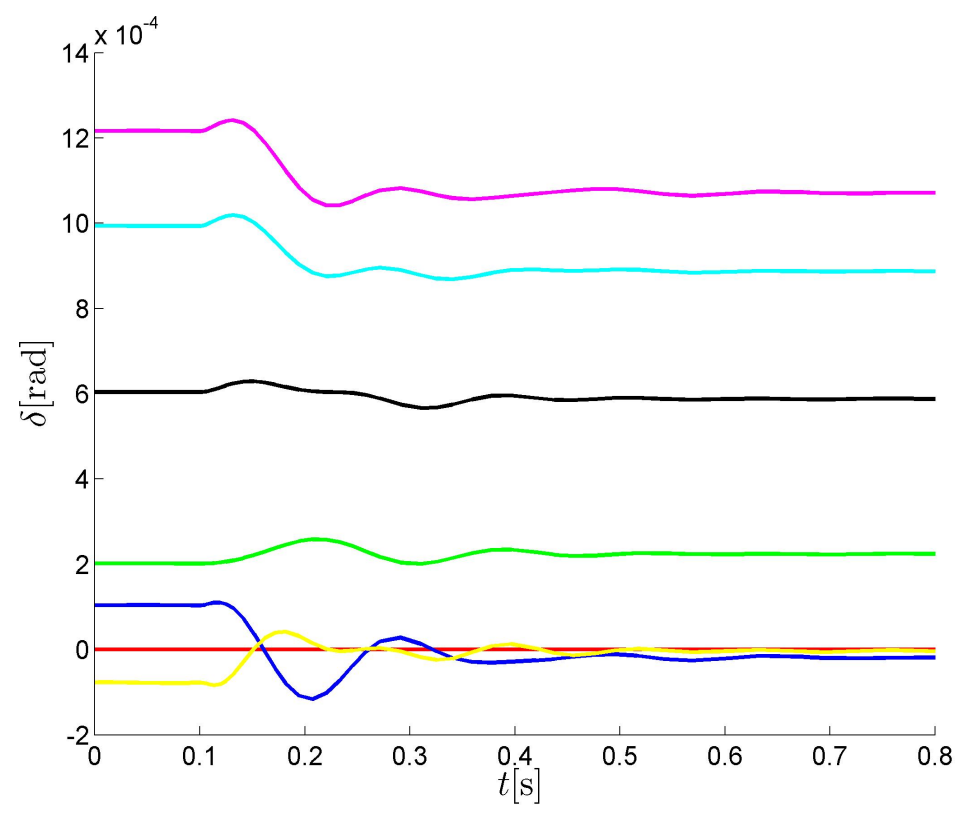

Figure 5.12: Step responses of terminal voltage angles of seven inverters.

\footnotetext{
4 The colors of Figs. 5.12 - 5.14 are denoted as red: Inv1, blue: Inv2, green: Inv3, black: Inv4, magenta: Inv5, cyan: Inv6, yellow: Inv7.

5 Colors and line styles in Figs. 5.15-5.19 are denoted as 13th-order: dashed black line, ninth-order: solid red line, fifth-order: dotted green line and third-order: solid blue line.
} 


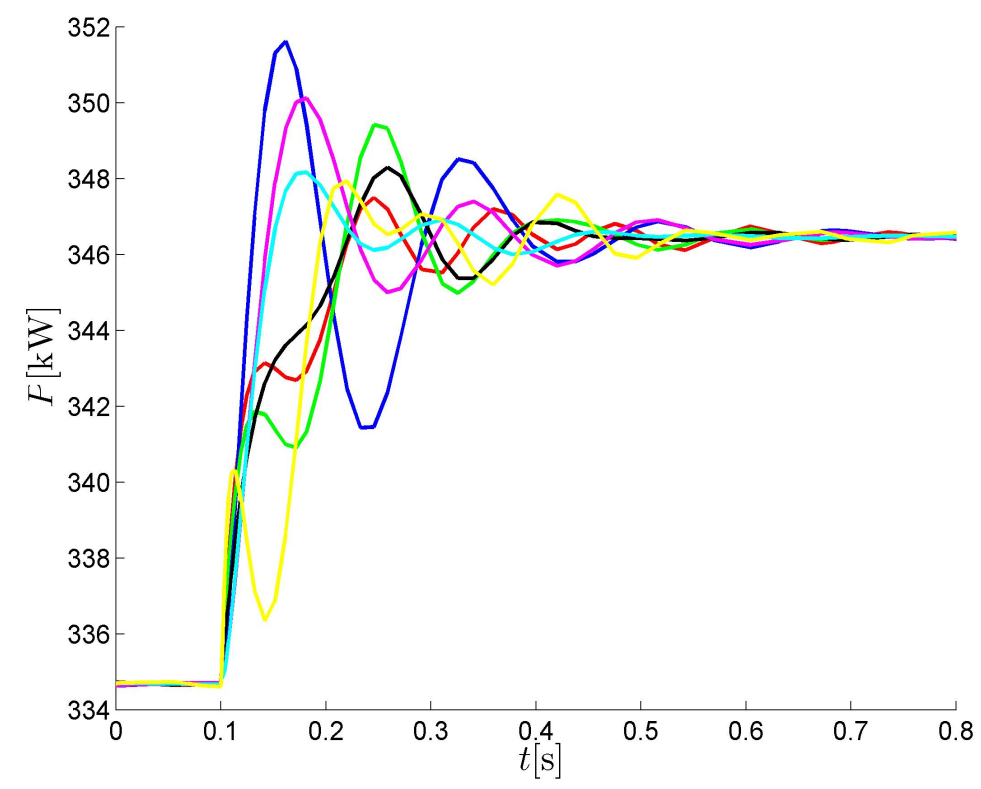

Figure 5.13: Step responses of active power of seven inverters.

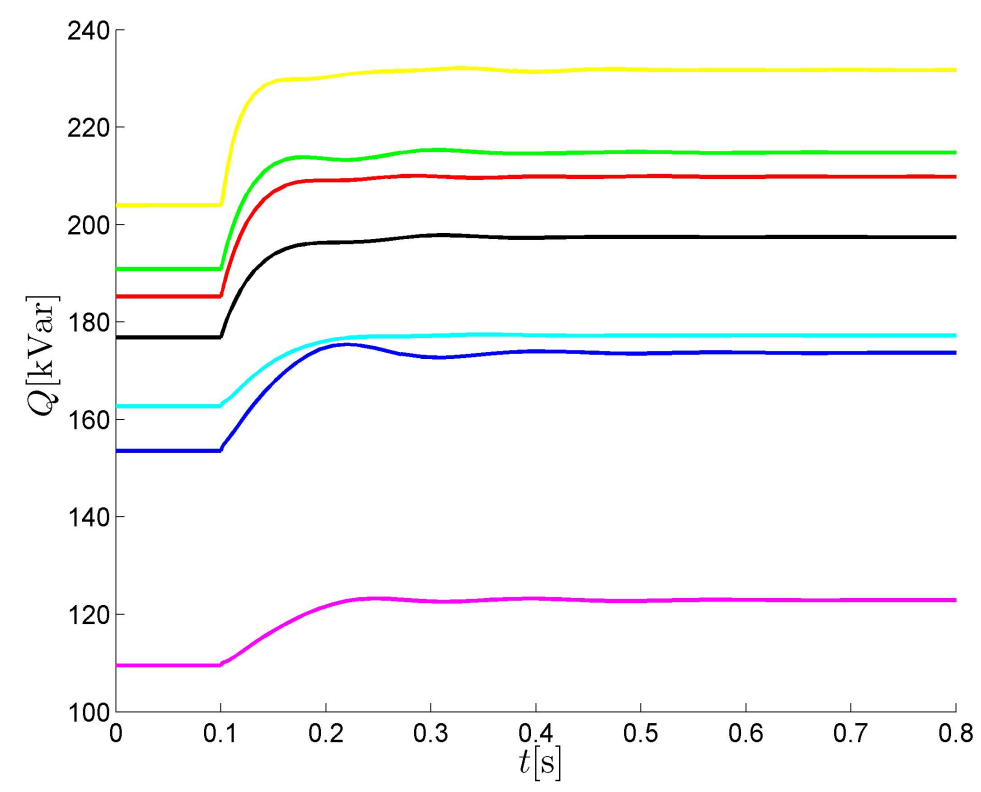

Figure 5.14: Step responses of reactive power of seven inverters. 


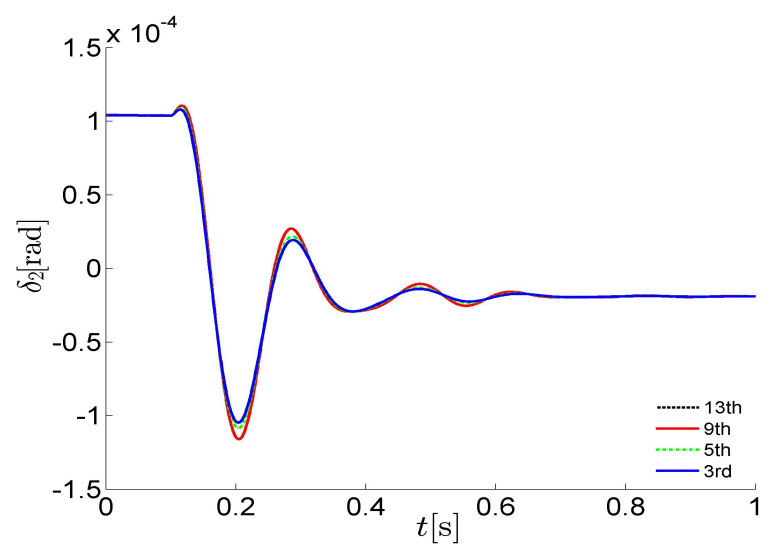

(a)

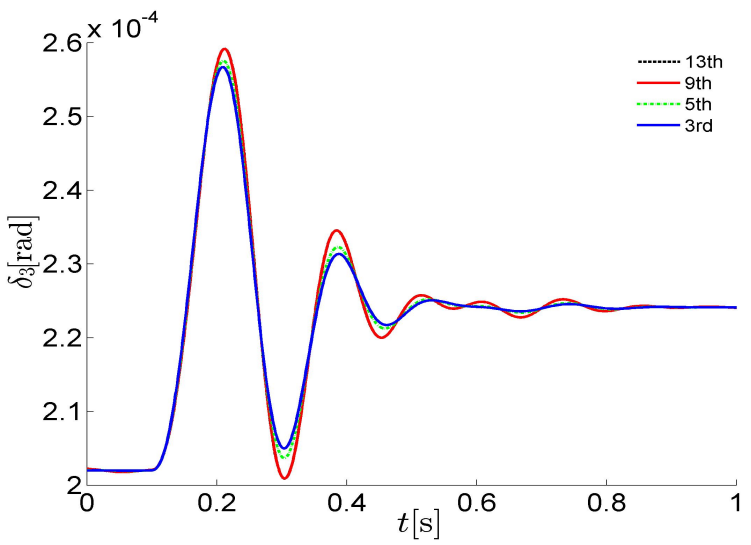

(b)

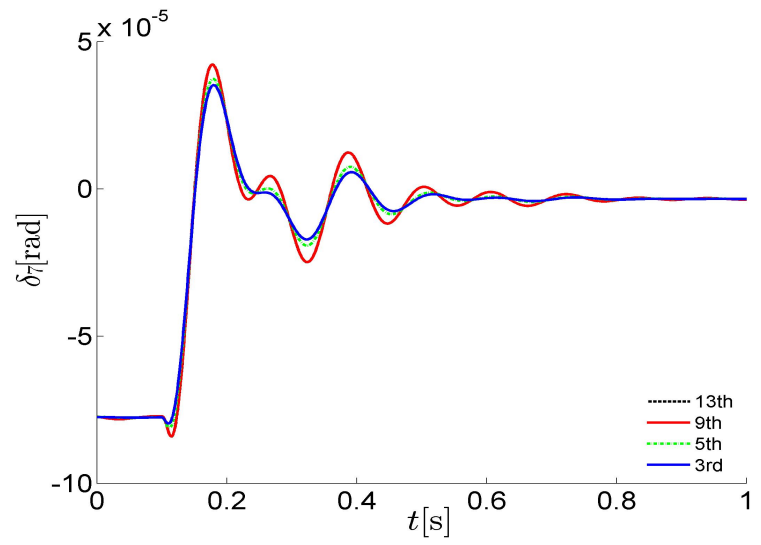

(c)

Figure 5.15: Step responses of terminal voltage angle comparing the original 13th-order and the spatio-temporal reduced models: (a) $\delta_{2} ;(\mathrm{b}) \delta_{3} ;(\mathrm{c}) \delta_{7}$. 


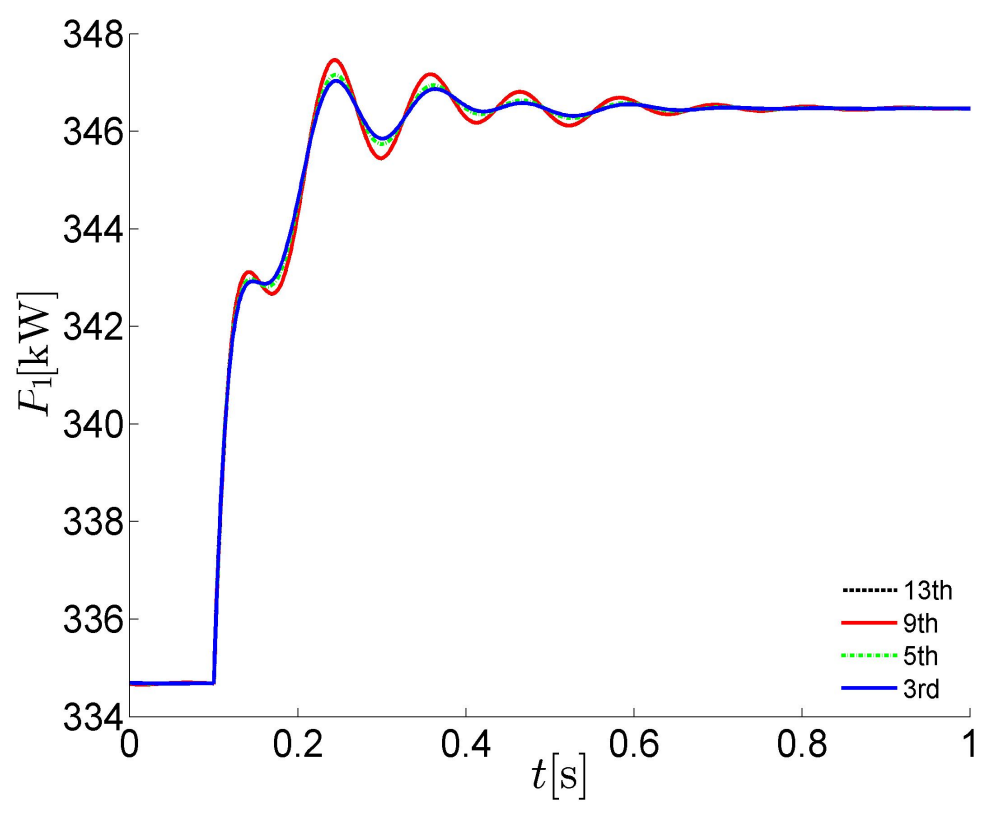

(a)

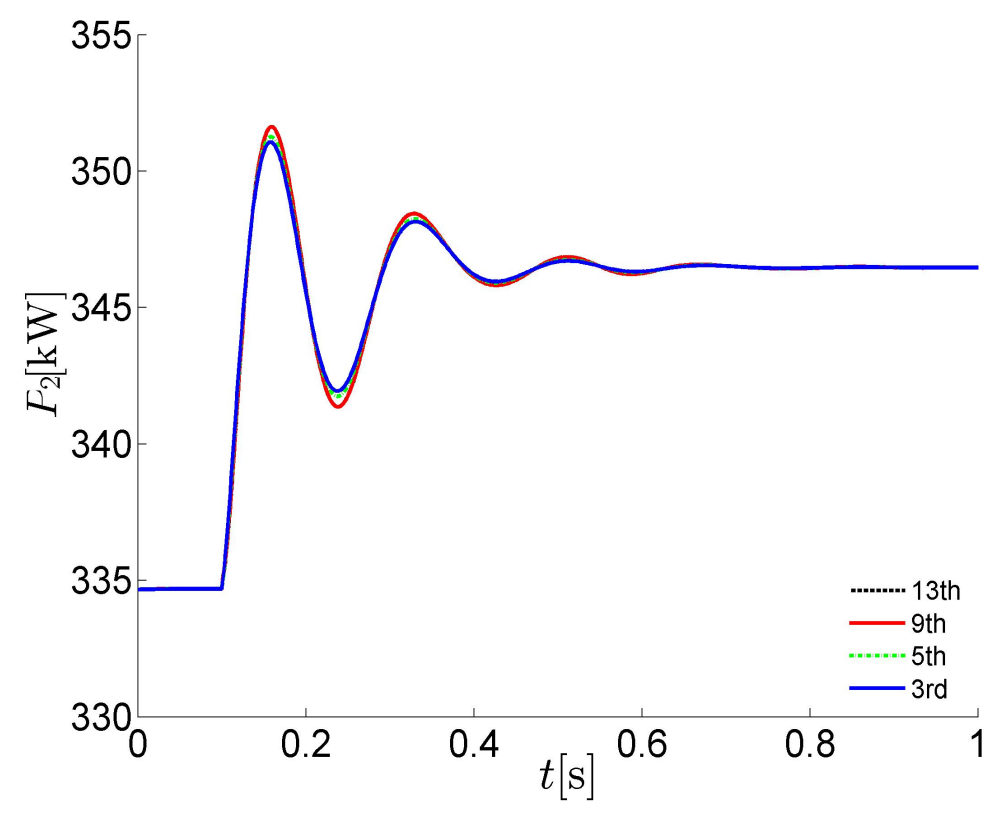

(b)

Figure 5.16: Step responses of active power comparing the original 13th-order and the spatio-temporal reduced models: (a) $P_{1}$; (b) $P_{2}$. 


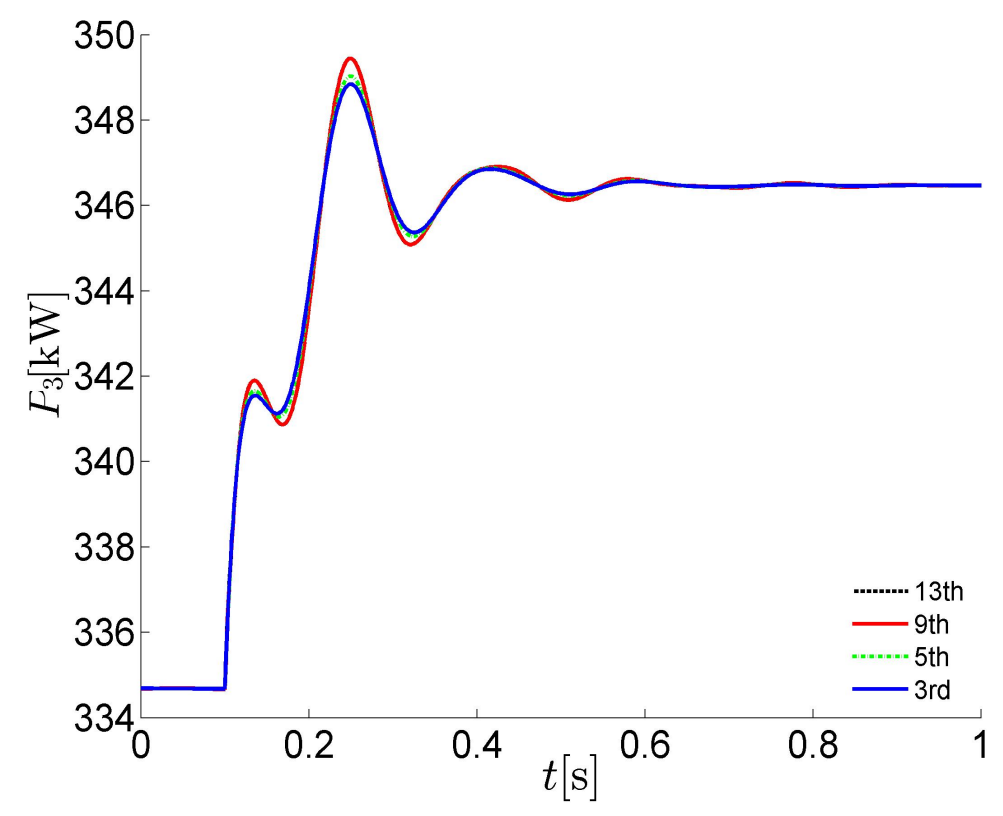

(a)

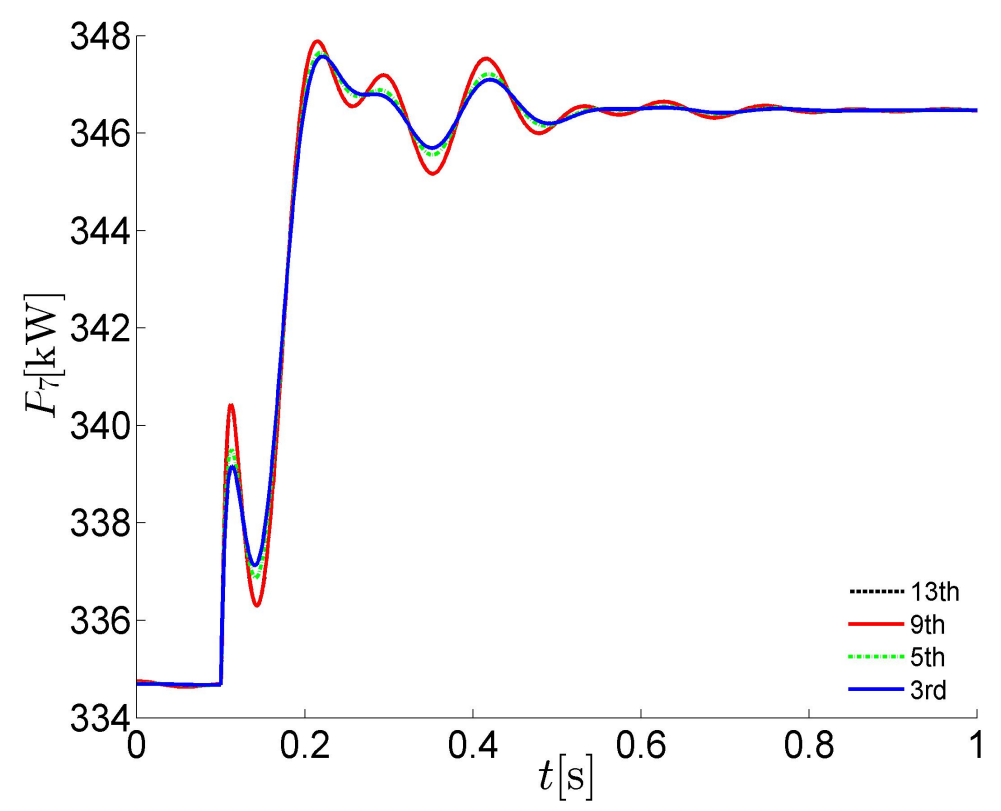

(b)

Figure 5.17: Step responses of active power comparing the original 13th-order and the spatio-temporal reduced models: (a) $P_{3} ;(\mathrm{b}) P_{7}$. 


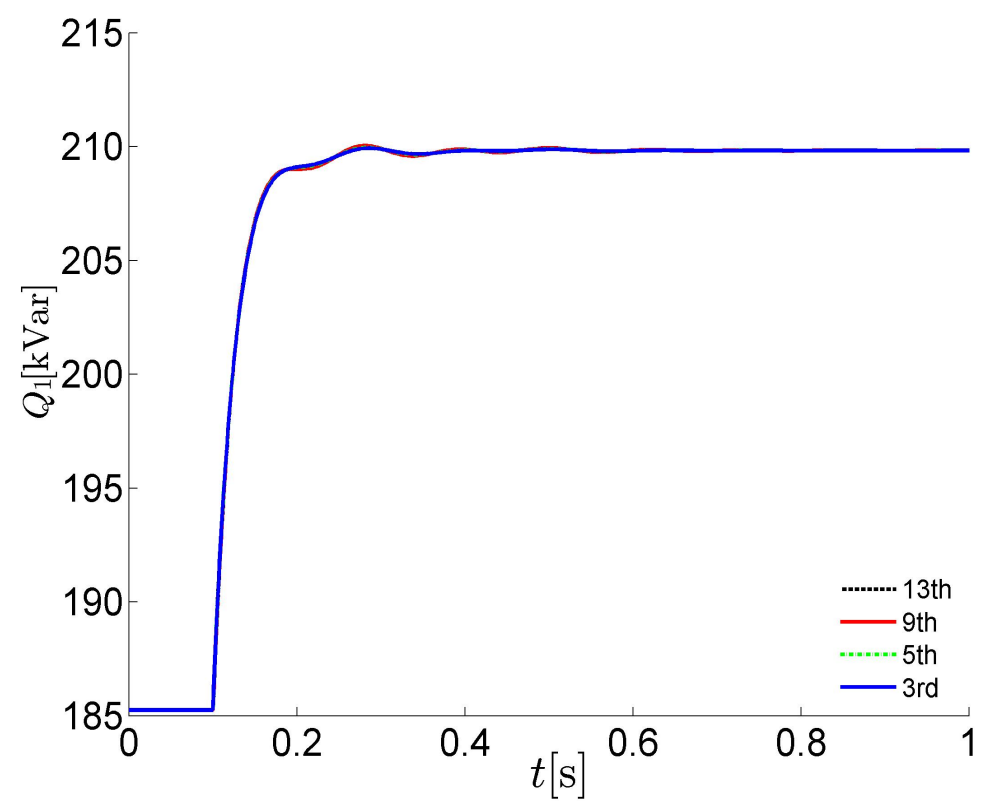

(a)

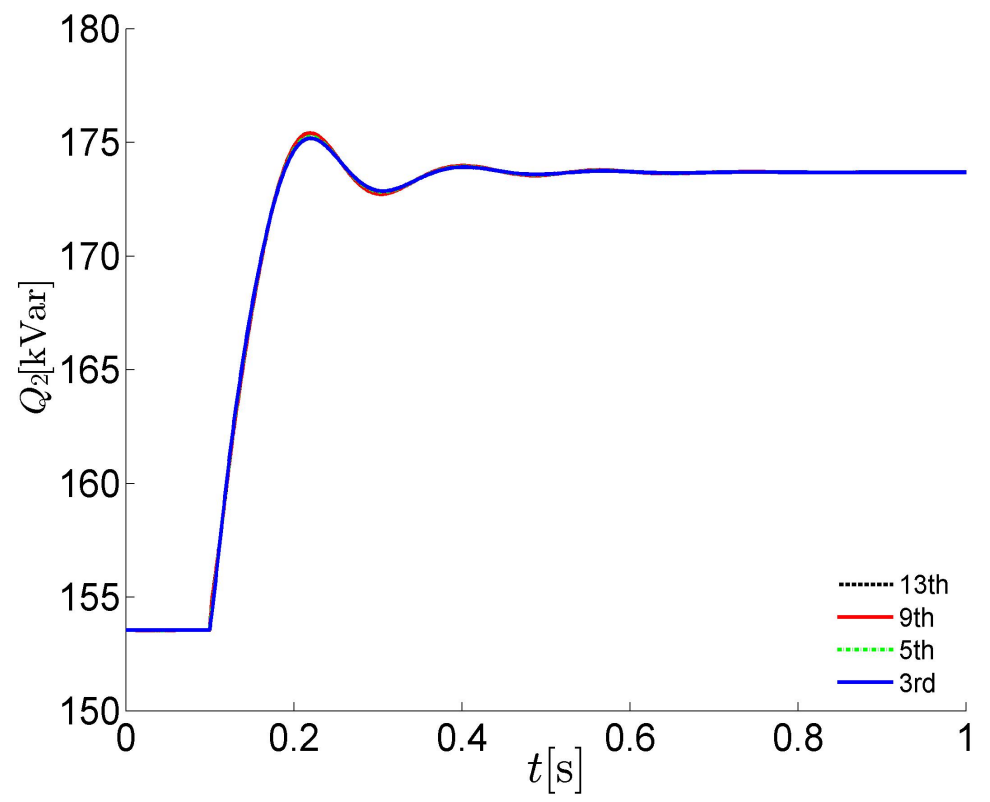

(b)

Figure 5.18: Step responses of reactive power comparing the original 13th-order and the spatio-temporal reduced models: (a) $Q_{1}$; (b) $Q_{2}$. 


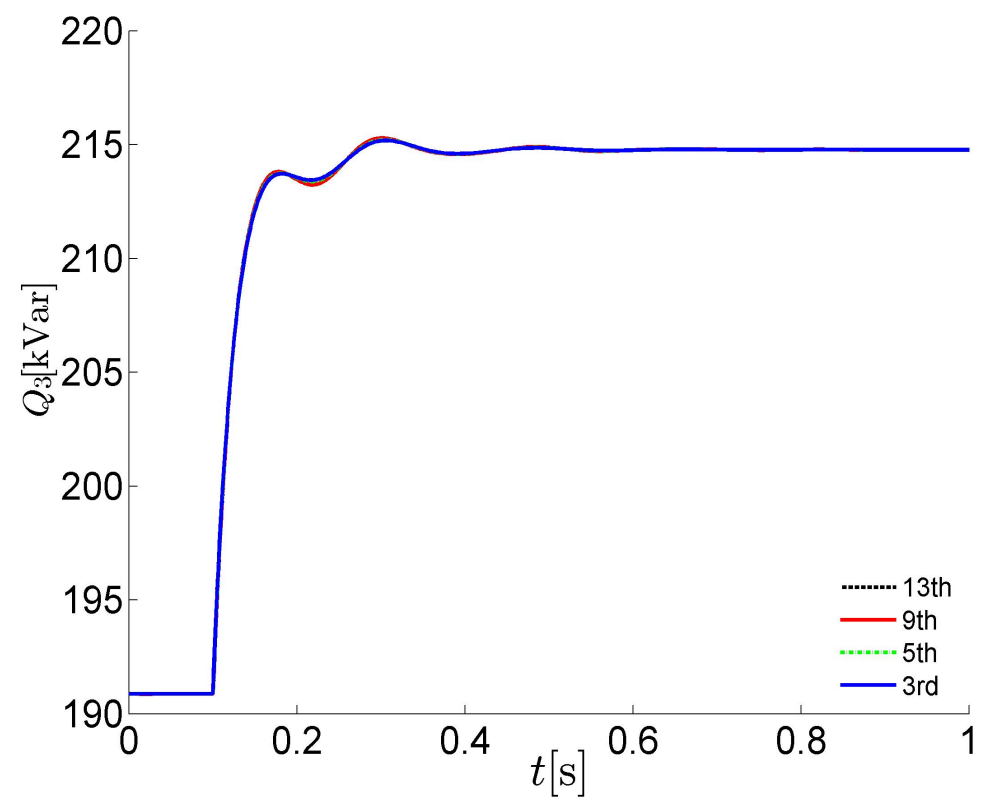

(a)

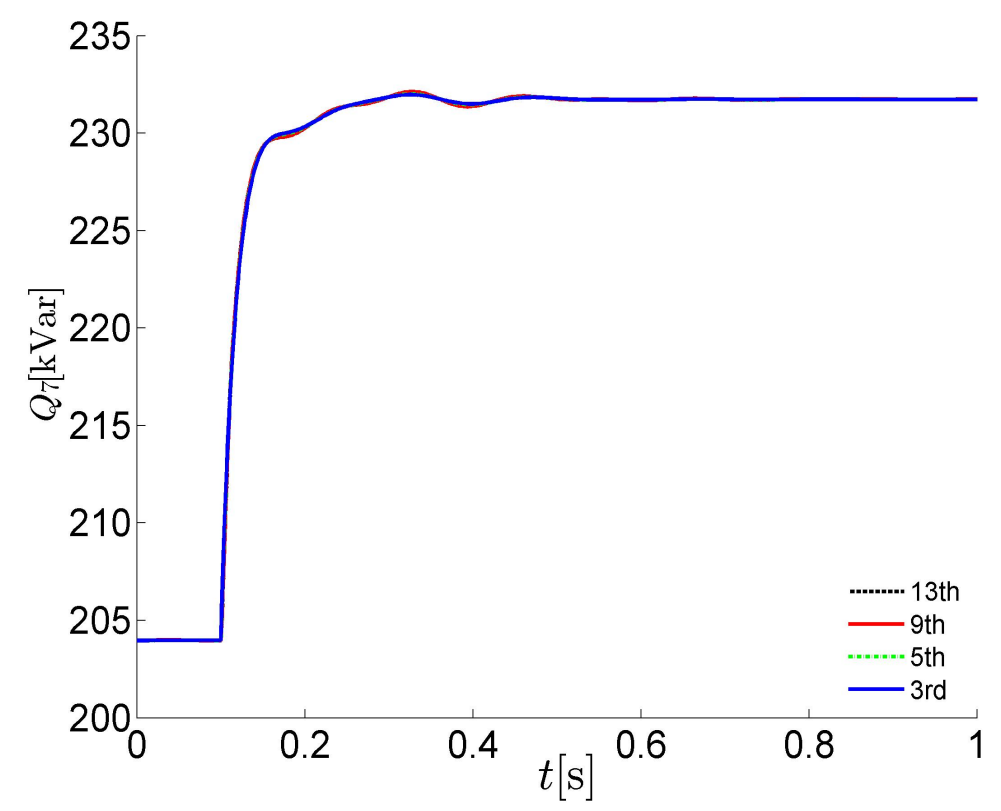

(b)

Figure 5.19: Step responses of reactive power comparing the original 13th-order and the spatio-temporal reduced models: (a) $Q_{3} ;$ (b) $Q_{7}$. 


\subsubsection{Systematic Design of Droop Coefficients}

In most of the studied droop-controlled inverters, equally rated inverters with the same droop coefficients were employed [4, 7]. However, loads are practically distributed unequally. In this case, equivalent loads cannot be balanced by the electrical closest sources which means power flows through between inverters. And this causes power losses and voltage deviations. When locally balanced loads against supplies, the power flow between inverters will be minimized which reducing the power losses and voltage deviations significantly. Thus, we propose an systematical droop coefficients design method based on Kron-reduced network to minimize power losses and voltage deviations.

Figure 5.20 shows the Kron-reduced network model of the modified IEEE 37-bus testing system of figure 5.11. The equivalent loads that the $i^{\text {th }}$ inverter should support in the microgrid can be approximately calculated by

$$
S_{\mathrm{eq}}^{i}=\frac{V_{\mathrm{nom}}\left(V_{\mathrm{nom}}\right)^{*}}{\left(Z_{\mathrm{eq}}^{i}\right)^{*}}
$$

where $V_{\text {nom }}^{i}\left(Z_{\text {eq }}^{i}\right)$ is the nominal voltage (equivalent impedance of the load) of the $i^{t h}$ bus. Let $P_{\text {eq }}^{i}\left(Q_{\text {eq }}^{i}\right)$ denote the real (imaginary) part of $S_{\text {eq }}^{i}$. To balance equivalent loads against available energy supply locally, we can pick $S_{\text {eq }}^{i}$ as the maximum power when design droop coefficients of the $i^{t h}$ inverter.

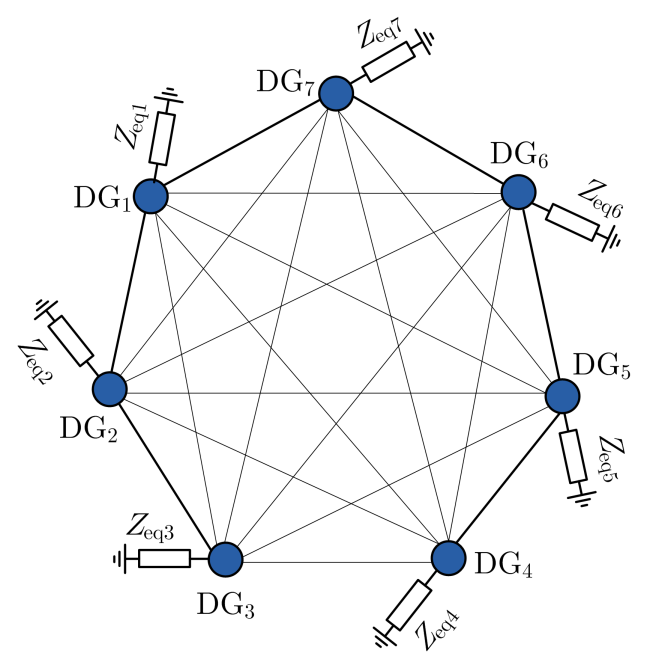

Figure 5.20: Oneline diagram of IEEE 37-bus system after Kron reduction 
Furthermore, to mimic the dynamic behavior of synchronous machines, all units in service should adjust their active power outputs to achieve the same frequency increments (or decrements) when loads decrease (or increase). Assuming: (1) the nominal frequency and magnitude of terminal voltage of all inverters are the same; (2) minimum active (reactive) power $P_{\min }\left(Q_{\min }\right)$ are zeros, and picking $0.05 \%(2 \%)$ as frequency (voltage) deviation limits in droop controls, then from (2.1) we have

$$
m_{\mathrm{P}}^{i}=\frac{0.05 \% \omega_{\mathrm{nom}}}{P_{\max }^{i}}, \quad n_{\mathrm{Q}}^{i}=\frac{2 \% V_{\mathrm{nom}}}{Q_{\max }^{i}} .
$$

When load changes, we denote $\Delta P_{i}\left(\Delta \omega^{i}\right)$ as active power (frequency) variation of the $i^{\text {th }}$ inverter. From (2.5), we have

$$
\omega^{i}+\Delta \omega^{i}=\omega_{\mathrm{nom}}-m_{\mathrm{P}}^{i}\left(P^{i}+\Delta P^{i}\right)
$$

In (5.2), (5.3) and notice that $\omega^{i}=\omega_{\text {nom }}-m_{\mathrm{P}}^{i} P^{i}$, we obtain

$$
\Delta \omega^{i}=-m_{\mathrm{P}}^{i} \Delta P^{i}=-\frac{0.05 \% \omega_{\mathrm{nom}}}{P_{\max }^{i}} \Delta P^{i}
$$

From (5.4), $\Delta P^{i}$ is proportional to its maximum capacity $P_{\max }^{i}$ in steady state when frequency deviation of all inverters are the same. A similar analysis can be employed for voltage deviation and maximum reactive power.

Based on the equivalent loads obtained from Kron reduction in (5.1) and the conclusion that $\Delta P^{i}$ is proportional to $P_{\max }^{i}$ in steady state, we let

$$
\begin{gathered}
P_{\max }^{i}=P_{\mathrm{eq}}^{i}, \\
Q_{\max }^{i}=Q_{\text {eq }}^{i} .
\end{gathered}
$$

and design droop coefficients by (5.2). To verify the systematically designed droop coefficients, we build two scenarios for microgrid shown in Fig. 5.11. The equivalent loads and droop coefficients of the two scenarios are listed in Table 5.2 and 5.3 . Notice that scenario 1 has been discussed in Subsection 5.2.1. 
Table 5.2: Maximum capacity of the inverters

\begin{tabular}{cccccc}
\hline inverter & bus & \multicolumn{2}{c}{ Scenario 1 } & \multicolumn{2}{c}{ Scenario 2 } \\
name & number & $P(\mathrm{~kW})$ & $Q(\mathrm{kVar})$ & $P(\mathrm{~kW})$ & $Q(\mathrm{kVar})$ \\
\hline Inv 1 & 718 & 339.19 & 171.13 & 362.42 & 175.5 \\
Inv 2 & 724 & 339.19 & 171.13 & 249.02 & 120.25 \\
Inv 3 & 729 & 339.19 & 171.13 & 386.68 & 196.89 \\
Inv 4 & 731 & 339.19 & 171.13 & 342.75 & 204.72 \\
Inv 5 & 736 & 339.19 & 171.13 & 154.11 & 72.51 \\
Inv 6 & 741 & 339.19 & 171.13 & 347.28 & 186.4 \\
Inv 7 & 742 & 339.19 & 171.13 & 479.25 & 214.42 \\
\hline \multicolumn{2}{c}{ total } & 2374.3 & 1197.9 & 2374.3 & 1197.9 \\
\hline
\end{tabular}

Table 5.3: Droop coefficients

\begin{tabular}{cccccc}
\hline inverter & bus & \multicolumn{2}{c}{ Scenario 1} & \multicolumn{2}{c}{ Scenario 2 } \\
name & number & $m_{\mathrm{P}}\left(\times 10^{-7}\right)$ & $n_{\mathrm{Q}}\left(\times 10^{-4}\right)$ & $m_{\mathrm{P}}\left(\times 10^{-7}\right)$ & $n_{\mathrm{Q}}\left(\times 10^{-4}\right)$ \\
\hline Inv 1 & 718 & 4.631 & 5.61 & 4.141 & 5 \\
Inv 2 & 724 & 4.631 & 5.61 & 6.111 & 8 \\
Inv 3 & 729 & 4.631 & 5.61 & 3.889 & 5 \\
Inv 4 & 731 & 4.631 & 5.61 & 4.421 & 5 \\
Inv 5 & 736 & 4.631 & 5.61 & 9.924 & 13 \\
Inv 6 & 741 & 4.631 & 5.61 & 4.414 & 5 \\
Inv 7 & 742 & 4.631 & 5.61 & 3.094 & 4 \\
\hline
\end{tabular}

Table 5.4: Power losses and voltage deviations

\begin{tabular}{ccc}
\hline items & power losses(W) & max voltage deviation(V) \\
\hline Scenario 1 & 777.5752 & 31.1662 \\
Scenario 1 (load step) & 1215.0 & 41.4916 \\
Scenario 2 & 5.3112 & 4.8031 \\
Scenario 2 (load step) & 67.3823 & 5.7616 \\
\hline
\end{tabular}


To test the designed coefficients with load steps, we applied a step change in load at bus 701 by changing the load impedance from $R_{1}=87.77 \Omega$ to $47.77 \Omega$. The step responses of the terminal voltage angle, active power and reactive power of Scenario 1 are shown in Figs. 5.12 - 5.14, and Scenario 2 are shown in Figs. 5.21-5.23. 6

With the designed coefficients, when load changes occur, each inverter contributes different active powers (reactive powers) to support the frequency (voltage) of the inverters. The calculated power losses and maximum voltage deviations before and after the load step are shown in Table 5.4 (notice the total active power load is $2.374 \mathrm{MW}$ ). Compared to inverters with equal droop coefficients, the power loss and maximum voltage deviations of the microgrid are greatly reduced before and after load step.

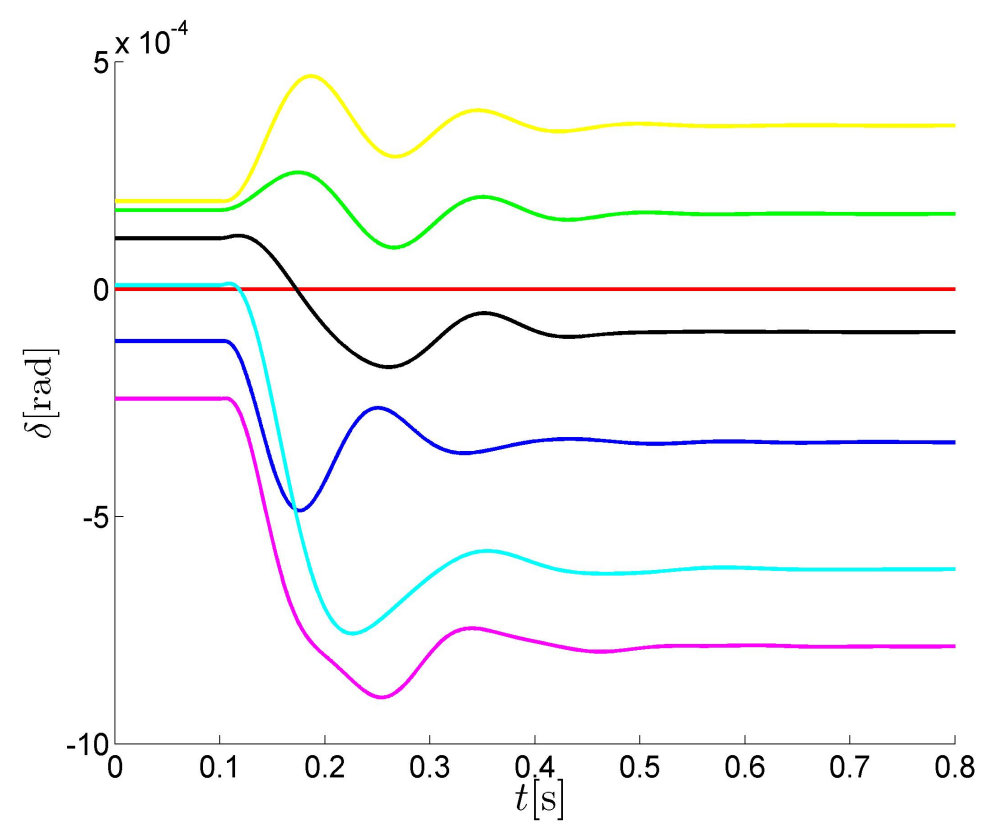

Figure 5.21: Scenario 2: step responses of terminal voltage angles of seven inverters.

\footnotetext{
6 The colors of plots indicate red: Inv1; blue: Inv2; green: Inv3; black: Inv4; magenta: Inv5; cyan: Inv6; yellow: Inv7.
} 


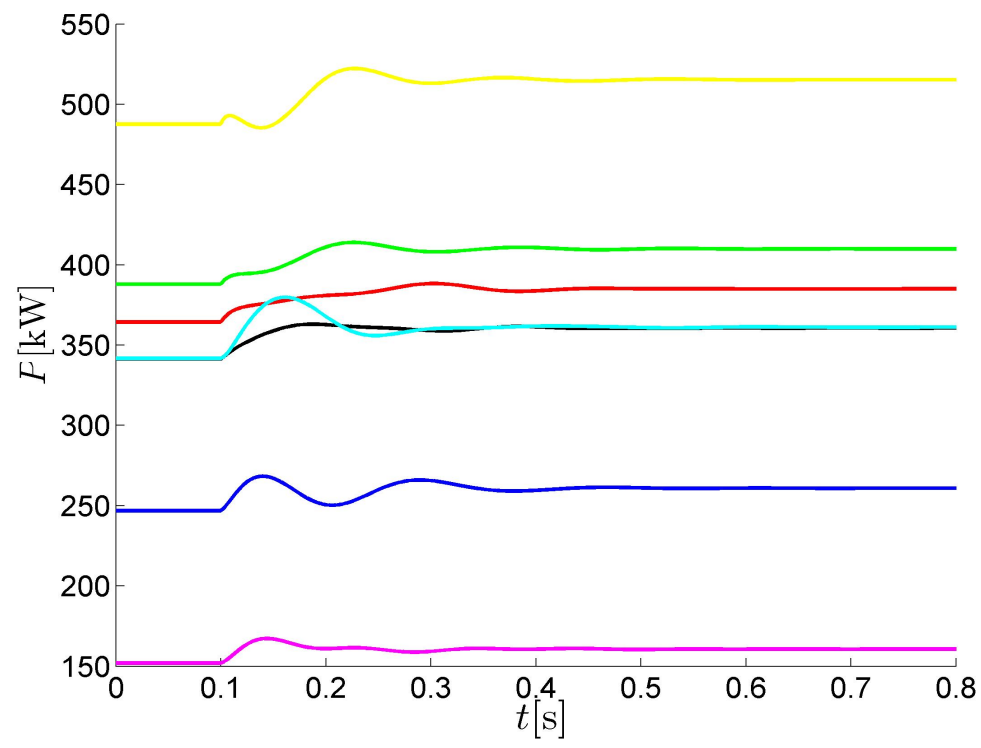

Figure 5.22: Scenario 2: step responses of active power of seven inverters.

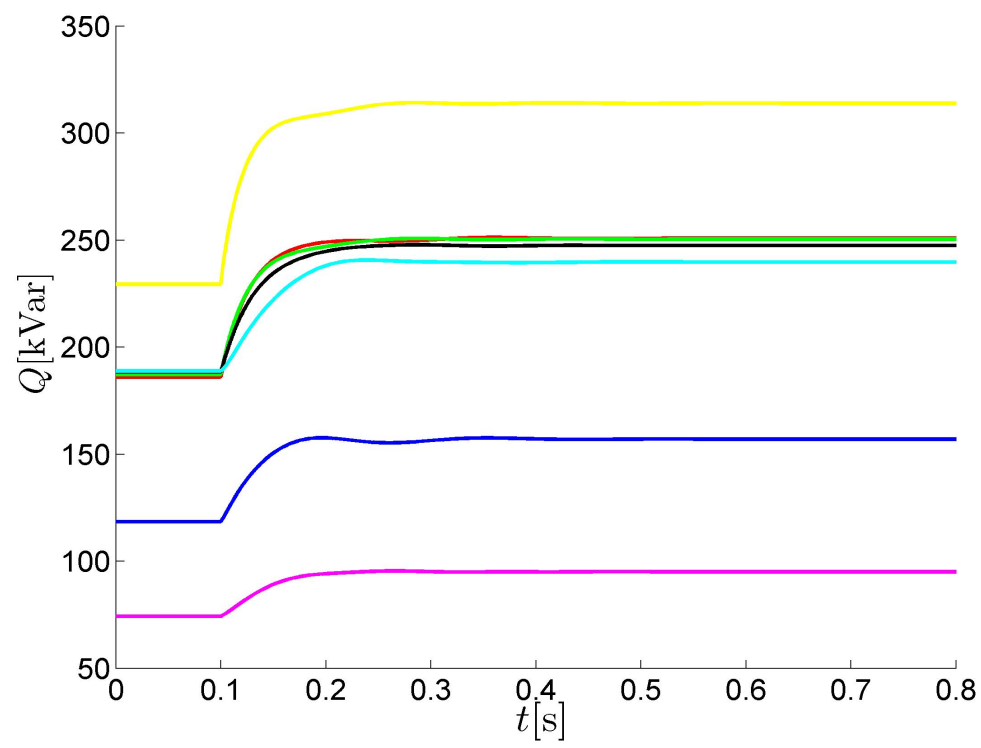

Figure 5.23: Scenario 2: step responses of reactive power of seven inverters. 


\subsection{Comparison of Simulation Speed Between the Full- order and the Reduced-Order Models}

The dynamic models of the inverter-based islanded microgrid models are programmed in MATLAB (Version 7.8.0). To compare the simulation speed by using different order of spatio-temporal reduced model, the models are tested in a personal computer with the following configuration:

- Processor: Intel(R) Core(TM) i7-2600K CPU @ 3.4GHz

- Installed RAM: 24.0 GB

- System type: 64-bit Operating System

To make the simulation result comparable, we use the same functions in MATLAB and the same simulation parameters for different order models. The total simulation time is two seconds. In the first second, the models simulated from start up to steady state; when $t=1.0$ s we apply a load step and simulate until $t=2.0 \mathrm{~s}$.

Table 5.5: Test of computational time in six-bus system

\begin{tabular}{ccc}
\hline model order & simulation time(from start up) & step response time \\
\hline 13th-order & $0.9472 \mathrm{~s}$ & $0.3359 \mathrm{~s}$ \\
ninth-order & $0.7991 \mathrm{~s}$ & $0.2951 \mathrm{~s}$ \\
fifth-order & $0.6633 \mathrm{~s}$ & $0.2602 \mathrm{~s}$ \\
third-order & $0.3653 \mathrm{~s}$ & $0.0955 \mathrm{~s}$ \\
\hline
\end{tabular}

Table 5.6: Test of computational time in IEEE 37-bus system

\begin{tabular}{ccc}
\hline model order & simulation time(from start up) & step response time \\
\hline 13 th-order & $2.37 \mathrm{~s}$ & $1.3 \mathrm{~s}$ \\
ninth-order & $1.79 \mathrm{~s}$ & $1.19 \mathrm{~s}$ \\
fifth-order & $1.26 \mathrm{~s}$ & $0.93 \mathrm{~s}$ \\
third-order & $0.67 \mathrm{~s}$ & $0.33 \mathrm{~s}$ \\
\hline
\end{tabular}


Tables 5.5 and 5.6 show that compared to the thirteenth-order model [4], the spatiotemporal reduced third-order model can reduce the simulation time to approximately one fourth for the modified IEEE 37-bus system, and reduced to approximately one third compared to the ninth-order reduced model. In conclusion, the spatio-temporal reducedorder models of the inverter-based islanded microgrids derived in previous chapters are verified by the simulations to be

- accurately capture relevant dynamic behaviors of the original full-order model;

- can significantly reduce the computational time in the six-bus microgrid and IEEE 37-bus system;

- provide a systematical droop coefficients design strategy that minimizes power losses and voltage deviations in steady state. 


\section{Chapter 6}

\section{Conclusions and Future Work}

\subsection{Conclusions}

In this thesis, model reduction methods are proposed for systematically reducing largesignal dynamic models of inverter-based islanded microgrids in both spatial and temporal aspects. The reduced-order models are verified by numerical simulations to accurately describe the original dynamics of the system with significantly reduced computational burden. In addition, spatial model reduction method based on Kron reduction is employed to isolate the mutual inverter interactions and clearly illustrate the equivalent loads that the inverters have to support in the microgrid. Based on the Kron-reduced network model, a systematical droop coefficients design strategy is proposed to minimize the power losses and voltage deviations.

One contribution of the thesis is the derivation of the spatio-temporal reduced fifth, third- and single-order models from the original large-signal dynamic model of the inverter-based islanded microgrid, and the development of software package in MATLAB for dynamic simulation of microgrids with different order of inverter models and any number of buses. The other contribution is the proposed control design strategy which provides a reference for choosing droop coefficients to minimize power losses and voltage deviations in steady state. 


\subsection{Future Work}

The models proposed in this work are expected to aid future efforts in modeling, analysis, and control of microgrids. In particular, the following awareness of future work are envisioned:

- In this thesis, only impedance loads and current loads were studied. Constant power loads could be modeled in this framework.

- We mainly focus on modeling islanded microgrids. Grid connected operation should be investigated as well.

- Design of sparse control architectures for secondary-level control. 


\section{References}

[1] Michael Burr, Michael Zimmer, Brian Meloy, James Bertrand, Walter Levesque, Guy Warner, and John McDonald. Minnesota microgrids: barriers, opportunities, and pathways toward energy assurance. Technical report, Minnesota Department of Commerce, 092013.

[2] P. Basak, A. K. Saha, S. Chowdhury, and S. P. Chowdhury. Microgrid: Control techniques and modeling. In Universities Power Engineering Conference (UPEC), 2009 Proceedings of the 44th International, pages 1-5, 2009.

[3] M. Strelec, K. Macek, and A. Abate. Modeling and simulation of a microgrid as a stochastic hybrid system. In Innovative Smart Grid Technologies (ISGT Europe), 2012 3rd IEEE PES International Conference and Exhibition on, pages 1-9, 2012.

[4] N. Pogaku, M. Prodanovic, and T.C. Green. Modeling, analysis and testing of autonomous operation of an inverter-based microgrid. Power Electronics, IEEE Transactions on, 22(2):613-625, march 2007.

[5] G. S. Ladde, D.D.Ladde D. D. Siljak, G.S., and D D DLadde. Multiparameter singular perturbations of linear systems with multiple time scales. Automatica, 19(4):385-394, 1983.

[6] Sungwoo Bae and A. Kwasinski. Dynamic modeling and operation strategy for a microgrid with wind and photovoltaic resources. Smart Grid, IEEE Transactions on, 3(4):1867-1876, dec. 2012.

[7] A. Bidram and A. Davoudi. Hierarchical structure of microgrids control system. Smart Grid, IEEE Transactions on, 3(4):1963-1976, 2012. 
[8] A. Bidram, A. Davoudi, F.L. Lewis, and J.M. Guerrero. Distributed cooperative secondary control of microgrids using feedback linearization. Power Systems, IEEE Transactions on, $\mathrm{PP}(99): 1-9,2013$.

[9] D. Chaniotis and M. A. Pai. Model reduction in power systems using krylov subspace methods. Power Systems, IEEE Transactions on, 20(2):888-894, 2005.

[10] F.D. Freitas, J. Rommes, and N. Martins. Gramian-based reduction method applied to large sparse power system descriptor models. Power Systems, IEEE Transactions on, 23(3):1258-1270, 2008.

[11] J.H. Chow, R. Galarza, P. Accari, and W.W. Price. Inertial and slow coherency aggregation algorithms for power system dynamic model reduction. Power Systems, IEEE Transactions on, 10(2):680-685, 1995.

[12] E.H. Abed and R. Silva-Madriz. Stability of systems with multiple very small and very large parasitics. Circuits and Systems, IEEE Transactions on, 34(9):1107$1110,1987$.

[13] H. Khalil. Nonlinear Systems. Prentice Hall, Upper Saddle River, NJ, 2002.

[14] H. Khalil. Stability analysis of nonlinear multiparameter singularly perturbed systems. Automatic Control, IEEE Transactions on, 32(3):260-263, 1987.

[15] Guangyue Xu and V. Vittal. Slow coherency based cutset determination algorithm for large power systems. Power Systems, IEEE Transactions on, 25(2):877-884, 2010 .

[16] Guangyue Xu, V. Vittal, A. Meklin, and J.E. Thalman. Controlled islanding demonstrations on the wecc system. Power Systems, IEEE Transactions on, 26(1):334-343, 2011.

[17] Jr. P. V. Kokotovic, R. E. O'Malley and P. Sannuti. Singular perturbations and order reduction in control theory-an overview. Automatica, 12:123-132, 1976.

[18] H. K. Khalil. Asymptotic stability of nonlinear multiparameter singularly perturbed systems. Automatica, 17(1):797-804, 1978. 
[19] X. Xu, R. M. Mathur, J. Jiang, G.J. Rogers, and P. Kundur. Modeling of generators and their controls in power system simulations using singular perturbations. Power Systems, IEEE Transactions on, 13(1):109-114, 1998.

[20] J. H. Chow. Asymptotic stability of a class of nonlinear singularity perturbed systems. J. Franklin Inst., 305:123-132, 1978.

[21] Gabriel Kron. A method to solve very large physical systems in easy stages. Circuit Theory, Transactions of the IRE Professional Group on, PGCT-2:71-90, 1953.

[22] F. Dorfler and F. Bullo. Kron reduction of graphs with applications to electrical networks. Circuits and Systems I: Regular Papers, IEEE Transactions on, 60(1):150-163, 2013.

[23] R.M. Ciric, Antonio Padilha Feltrin, and L.F. Ochoa. Power flow in four-wire distribution networks-general approach. Power Systems, IEEE Transactions on, 18(4):1283-1290, 2003.

[24] J.M. Guerrero, J.C. Vasquez, J. Matas, L.G. de Vicuna, and M. Castilla. Hierarchical control of droop-controlled ac and dc microgrids - a general approach toward standardization. Industrial Electronics, IEEE Transactions on, 58(1):158$172,2011$.

[25] R. Majumder, B. Chaudhuri, A. Ghosh, R. Majumder, G. Ledwich, and F. Zare. Improvement of stability and load sharing in an autonomous microgrid using supplementary droop control loop. Power Systems, IEEE Transactions on, 25(2):796808, 2010.

[26] T.L. Vandoorn, B. Meersman, J.D.M. De Kooning, and L. Vandevelde. Transition from islanded to grid-connected mode of microgrids with voltage-based droop control. Power Systems, IEEE Transactions on, 28(3):2545-2553, 2013.

[27] Qing-Chang Zhong. Robust droop controller for accurate proportional load sharing among inverters operated in parallel. Industrial Electronics, IEEE Transactions on, 60(4):1281-1290, 2013. 
[28] Y.A. Mohamed and A.A. Radwan. Hierarchical control system for robust microgrid operation and seamless mode transfer in active distribution systems. Smart Grid, IEEE Transactions on, 2(2):352 -362, june 2011.

[29] H. Khalil. Stability analysis of nonlinear multiparameter singularly perturbed systems. In American Control Conference, 1987, pages 1219-1223, 1987.

[30] D. Peponides, P. V. Kokotovic, and J. H. Chow. Singular perturbations and time scales in nonlinear models of power system. Circuits and Systems, IEEE Transactions on, 29(11):758-767, 1982.

[31] Shengyi Su. Integrated feeder switching and voltage control for increasing distributed generation penetration. Master's thesis, National Sun Yat-Sen University, 2009 . 


\section{Appendix A}

\section{Detailed Models}

For the large-signal inverter-based islanded microgrids models, assume coordinate of inverter 1 as the common reference $D Q$-axis. The input of the inverter model $u=$ $\left[u^{1}, u^{2}, \ldots, u^{N}\right]^{\mathrm{T}} \in \mathbb{C}^{N \times 1}$ and the output current $i_{\mathrm{o}}=\left[i_{\mathrm{o}}^{1}, i_{\mathrm{o}}^{2}, \ldots, i_{\mathrm{o}}^{N}\right]^{\mathrm{T}} \in \mathbb{C}^{N \times 1}$.

\section{A.1 Single-Order Model}

The reduced spatio-temporal single-order large-signal dynamic model of inverter-based islanded microgrids includes $N$ inverters $(i=1,2, \ldots, N)$ is given by ( $N$ state variables)

$$
\begin{aligned}
\frac{d \delta^{i}}{d t} & =\omega_{\mathrm{nom}}^{i}-m_{\mathrm{P}}^{i} \frac{S^{i}+\left(S^{i}\right)^{*}}{2}-\omega_{\mathrm{com}}, \\
S^{i} & =\left(v_{\mathrm{o}}^{i}\right)^{\mathrm{ref}}\left(i_{\mathrm{o}}^{i}\right)^{*} \\
i_{\mathrm{o}}^{i} & =\frac{\left(v_{\mathrm{o}}^{i}\right)^{\mathrm{ref}}-u^{i}}{R_{\mathrm{c}}^{i}} \\
\left(v_{\mathrm{o}}^{i}\right)^{\mathrm{ref}} & =v_{\mathrm{nom}}^{i}-n_{\mathrm{Q}}^{i} \frac{S^{i}-\left(S^{i}\right)^{*}}{2}, \\
\omega_{\mathrm{com}} & =\omega_{\mathrm{nom}}^{1}-m_{\mathrm{P}}^{1} \frac{S^{1}+\left(S^{1}\right)^{*}}{2}, \\
Y_{\mathrm{kr}} & =Y_{\alpha \alpha}-Y_{\alpha \beta} Y_{\beta \beta}^{-1} Y_{\alpha \beta}^{\mathrm{T}}, \\
u & =T^{-1} Y_{\mathrm{kr}}^{-1} T i_{\mathrm{o}} .
\end{aligned}
$$




\section{A.2 Third-Order Model}

The reduced spatio-temporal third-order large-signal dynamic model is given by $(3 \times N$ state variables)

$$
\begin{aligned}
\frac{d \delta^{i}}{d t} & =\omega_{\mathrm{nom}}^{i}-m_{\mathrm{P}}^{i} \frac{S^{i}+\left(S^{i}\right)^{*}}{2}-\omega_{\mathrm{com}}, \\
\frac{d S^{i}}{d t} & =-\omega_{\mathrm{c}}^{i} S^{i}+\omega_{\mathrm{c}}^{i}\left(v_{\mathrm{o}}^{i}\right)^{\mathrm{ref}}\left(i_{\mathrm{o}}^{i}\right)^{*}, \\
i_{\mathrm{o}}^{i} & =\frac{\left(v_{\mathrm{o}}^{i}\right)^{\mathrm{ref}}-u^{i}}{R_{\mathrm{c}}^{i}} \\
\left(v_{\mathrm{o}}^{i}\right)^{\mathrm{ref}} & =v_{\mathrm{nom}}^{i}-n_{\mathrm{Q}}^{i} \frac{S^{i}-\left(S^{i}\right)^{*}}{2}, \\
\omega_{\mathrm{com}} & =\omega_{\mathrm{nom}}^{1}-m_{\mathrm{P}} \frac{S^{1}+\left(S^{1}\right)^{*}}{2}, \\
Y_{\mathrm{kr}} & =Y_{\alpha \alpha}-Y_{\alpha \beta} Y_{\beta \beta}^{-1} Y_{\alpha \beta}^{\mathrm{T}}, \\
u & =T^{-1} Y_{\mathrm{kr}}^{-1} T i_{\mathrm{o}} .
\end{aligned}
$$

\section{A.3 Fifth-Order Model}

The reduced spatio-temporal fifth-order large-signal dynamic model is given by $(5 \times N$ state variables)

$$
\begin{aligned}
\frac{d \delta^{i}}{d t} & =\omega_{\mathrm{nom}}^{i}-m_{\mathrm{P}}^{i} \frac{S^{i}+\left(S^{i}\right)^{*}}{2}-\omega_{\mathrm{com}}, \\
\frac{d S^{i}}{d t} & =-\omega_{\mathrm{c}}^{i} S^{i}+\omega_{\mathrm{c}}^{i}\left(v_{\mathrm{o}}^{i}\right)^{\mathrm{ref}}\left(i_{\mathrm{o}}^{i}\right)^{*} \\
\frac{d i_{\mathrm{o}}^{i}}{d t} & =-\left(\frac{R_{\mathrm{c}}^{i}}{L_{\mathrm{c}}^{i}}+\mathrm{j} \omega^{i}\right) i_{\mathrm{o}}^{i}+\frac{\left(v_{\mathrm{o}}^{i}\right)^{\mathrm{ref}}-u^{i}}{L_{\mathrm{c}}^{i}}, \\
\left(v_{\mathrm{o}}^{i}\right)^{\mathrm{ref}} & =v_{\mathrm{nom}}^{i}-n_{\mathrm{Q}}^{i} \frac{S^{i}-\left(S^{i}\right)^{*}}{2} \\
\omega_{\mathrm{com}} & =\omega_{\mathrm{nom}}^{1}-m_{\mathrm{P}} \frac{S^{1}+\left(S^{1}\right)^{*}}{2} \\
\omega^{i} & =\omega_{\mathrm{com}}-m_{\mathrm{P}}^{i} \frac{S^{i}+\left(S^{1}\right)^{*}}{2} \\
Y_{\mathrm{kr}} & =Y_{\alpha \alpha}-Y_{\alpha \beta} Y_{\beta \beta}^{-1} Y_{\alpha \beta}^{\mathrm{T}}, \\
u & =T^{-1} Y_{\mathrm{kr}}^{-1} T i_{\mathrm{o}} .
\end{aligned}
$$




\section{A.4 Ninth-Order Model}

The ninth-order large-signal dynamic model of inverter-based islanded microgrids includes $N$ inverters $(i=1,2, \ldots, N)$ is given by $(9 \times N$ state variables $)$

$$
\begin{aligned}
\frac{d \delta^{i}}{d t} & =\omega_{\mathrm{nom}}^{i}-m_{\mathrm{P}}^{i} \frac{S^{i}+\left(S^{i}\right)^{*}}{2}-\omega_{\mathrm{com}}, \\
\frac{d S^{i}}{d t} & =-\omega_{\mathrm{c}}^{i} S^{i}+\omega_{\mathrm{c}}^{i}\left(v_{\mathrm{o}}^{i}\right)^{\mathrm{ref}}\left(i_{\mathrm{o}}^{i}\right)^{*}, \\
\frac{d i_{\mathrm{o}}^{i}}{d t} & =-\left(\frac{R_{\mathrm{c}}^{i}}{L_{\mathrm{c}}^{i}}+\mathrm{j} \omega^{i}\right) i_{\mathrm{o}}^{i}+\frac{\left(v_{\mathrm{o}}^{i}\right)^{\mathrm{ref}}-u^{i}}{L_{\mathrm{c}}^{i}}, \\
\frac{d \phi^{i}}{d t} & =\left(v_{\mathrm{o}}^{i}\right)^{\mathrm{ref}}-\left(v_{\mathrm{o}}\right)^{i}, \\
\frac{d \gamma^{i}}{d t} & =\left(i_{\mathrm{o}}^{i}\right)^{\mathrm{ref}}-\left(i_{\mathrm{o}}\right)^{i}, \\
\left(v_{\mathrm{o}}^{i}\right)^{\mathrm{ref}} & =v_{\mathrm{nom}}^{i}-n_{\mathrm{Q}}^{i} \frac{S^{i}-\left(S^{i}\right)^{*}}{2}, \\
\left(i_{o}^{i}\right)^{\mathrm{ref}} & =F^{i}\left(i_{o}\right)^{i}+\mathrm{j} \omega^{i}+K_{\mathrm{p}}^{\phi^{\mathrm{i}}} \frac{d \phi^{i}}{d t}+K_{\mathrm{i}}^{\phi^{\mathrm{i}}} \phi^{i}, \\
\left(v_{\mathrm{i}}^{i}\right)^{\mathrm{ref}} & =\mathrm{j} \omega_{\mathrm{nom}}^{i} L_{\mathrm{f}}^{i} i_{\mathrm{o}}^{i}+K_{\mathrm{p}}^{\gamma^{\mathrm{i}}} \frac{d \gamma^{i}}{d t}+K_{\mathrm{i}}^{\gamma^{\mathrm{i}}} \gamma^{i}, \\
\omega_{\mathrm{com}} & =\omega_{\mathrm{nom}}^{1}-m_{\mathrm{P}}^{1} \frac{S^{1}+\left(S^{1}\right)^{*}}{2}, \\
v_{\mathrm{o}}^{i} & =\frac{\left.\left(R_{\mathrm{c}}^{i} L_{\mathrm{f}}^{i}-R_{\mathrm{f}}^{i} L_{\mathrm{c}}^{i}\right) i_{\mathrm{o}}^{i}+L_{\mathrm{f}}^{i} v_{\mathrm{b}}^{i}+L_{\mathrm{c}}^{i} v_{\mathrm{i}}^{i}\right)^{\mathrm{ref}}}{L_{\mathrm{f}}^{i}+L_{\mathrm{c}}^{i}} \\
\omega^{i} & =\omega_{\mathrm{com}}-m_{\mathrm{P}}^{i} \frac{S^{i}+\left(S^{1}\right)^{*}}{2}, \\
Y_{\mathrm{kr}} & =Y_{\alpha \alpha}-Y_{\alpha \beta} Y_{\beta \beta}^{-1} Y_{\alpha \beta}^{\mathrm{T}}, \\
u & =T^{-1} Y_{\mathrm{kr}}^{-1} T i_{\mathrm{o}} .
\end{aligned}
$$




\section{Appendix B}

\section{Simulation Parameters}

\section{B.1 Six-Bus System}

Table B.1: Single inverter parameters

\begin{tabular}{cccc}
\hline Parameter & Value & Parameter & Value \\
\hline$f_{\mathrm{s}}$ & $8 \mathrm{kHz}$ & $\omega_{\mathrm{c}}$ & 31.41 \\
$m_{\mathrm{P}}$ & $9.4 \mathrm{e}-5$ & $n_{\mathrm{Q}}$ & $1.3 \mathrm{e}-3$ \\
$K_{\mathrm{p}}^{\phi}$ & 0.05 & $K_{\mathrm{i}}^{\phi}$ & 390 \\
$K_{\mathrm{p}}^{\gamma}$ & 10.5 & $K_{\mathrm{i}}^{\gamma}$ & $16 \mathrm{e} 3$ \\
$L_{\mathrm{f}}$ & $1.35 \mathrm{mH}$ & $R_{\mathrm{f}}$ & $0.1 \Omega$ \\
$L_{\mathrm{c}}$ & $0.35 \mathrm{mH}$ & $R_{\mathrm{c}}$ & $0.03 \Omega$ \\
$F$ & 0.75 & & \\
\hline
\end{tabular}

\section{B.2 IEEE 37-Bus System}

In order to simulate the microgrid, seven DG units are connected to buses: 718724729 731736741742. 
Table B.2: IEEE 37-bus system branch parameters

\begin{tabular}{|c|c|c|c|}
\hline From Node & To Node & Resistance(R) & Reactance $(\mathrm{X})$ \\
\hline 701 & 702 & 0.0866 & 0.0544 \\
\hline 702 & 705 & 0.1594 & 0.0590 \\
\hline 702 & 713 & 0.0885 & 0.0461 \\
\hline 702 & 703 & 0.1198 & 0.0746 \\
\hline 703 & 727 & 0.0958 & 0.0350 \\
\hline 703 & 730 & 0.1475 & 0.0765 \\
\hline 704 & 714 & 0.0323 & 0.0120 \\
\hline 704 & 720 & 0.1972 & 0.1023 \\
\hline 705 & 742 & 0.1281 & 0.0470 \\
\hline 705 & 712 & 0.0958 & 0.0350 \\
\hline 706 & 725 & 0.1115 & 0.0415 \\
\hline 707 & 724 & 0.3032 & 0.1124 \\
\hline 707 & 722 & 0.0479 & 0.0175 \\
\hline 708 & 733 & 0.0793 & 0.0406 \\
\hline 708 & 732 & 0.1281 & 0.0470 \\
\hline 709 & 731 & 0.1475 & 0.0765 \\
\hline 709 & 708 & 0.0793 & 0.0406 \\
\hline 710 & 735 & 0.0802 & 0.0295 \\
\hline 710 & 736 & 0.5106 & 0.1889 \\
\hline 711 & 741 & 0.0986 & 0.0516 \\
\hline 711 & 740 & 0.0802 & 0.0295 \\
\hline 713 & 704 & 0.1281 & 0.0664 \\
\hline 714 & 718 & 0.2074 & 0.0765 \\
\hline 720 & 707 & 0.3668 & 0.1364 \\
\hline 720 & 706 & 0.1475 & 0.0765 \\
\hline 727 & 744 & 0.0691 & 0.0359 \\
\hline 730 & 709 & 0.0488 & 0.0258 \\
\hline 733 & 734 & 0.1382 & 0.0719 \\
\hline 734 & 737 & 0.1576 & 0.0820 \\
\hline
\end{tabular}


Table B.3: IEEE 37-bus system branch parameters (continued)

\begin{tabular}{cccc}
\hline \hline From Node & To Node & Resistance(R) & Reactance(X) \\
\hline 734 & 710 & 0.2074 & 0.0765 \\
737 & 738 & 0.0986 & 0.0516 \\
738 & 711 & 0.0986 & 0.0516 \\
744 & 728 & 0.0802 & 0.0295 \\
744 & 729 & 0.1115 & 0.0415 \\
775 & 709 & 0.0276 & 0.0184 \\
\hline
\end{tabular}


Table B.4: IEEE 37-bus system load parameters

\begin{tabular}{|c|c|c|}
\hline Node Number & Resistance $(\mathrm{R})$ & Reactance $(\mathrm{X})$ \\
\hline 701 & 87.77 & 43.89 \\
\hline 712 & 666.85 & 313.40 \\
\hline 713 & 666.85 & 313.40 \\
\hline 714 & 1483.14 & 700.69 \\
\hline 718 & 666.85 & 313.40 \\
\hline 720 & 666.85 & 313.40 \\
\hline 722 & 344.01 & 171.04 \\
\hline 724 & 1316.57 & 658.29 \\
\hline 725 & 1316.57 & 658.29 \\
\hline 727 & 1316.57 & 658.29 \\
\hline 728 & 438.86 & 219.43 \\
\hline 729 & 1316.57 & 658.29 \\
\hline 730 & 666.85 & 313.40 \\
\hline 731 & 666.85 & 313.40 \\
\hline 732 & 1316.57 & 658.29 \\
\hline 733 & 352.64 & 1657.29 \\
\hline 734 & 1316.57 & 658.29 \\
\hline 735 & 666.85 & 313.40 \\
\hline 736 & 1316.57 & 658.29 \\
\hline 737 & 395.03 & 197.09 \\
\hline 738 & 441.36 & 217.53 \\
\hline 740 & 666.85 & 313.40 \\
\hline 741 & 1316.57 & 658.29 \\
\hline 742 & 606.78 & 287.73 \\
\hline 744 & 1316.57 & 658.29 \\
\hline
\end{tabular}

